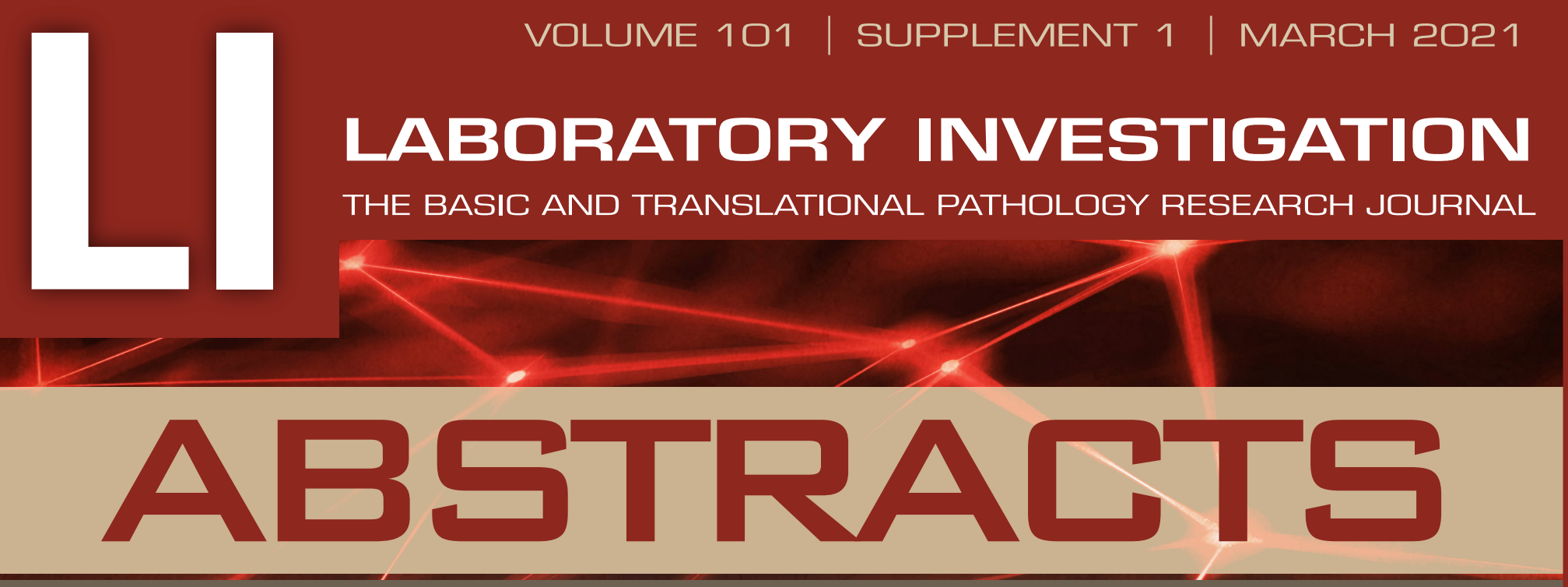

HEAD AND NECK PATHOLOGY (635-663)

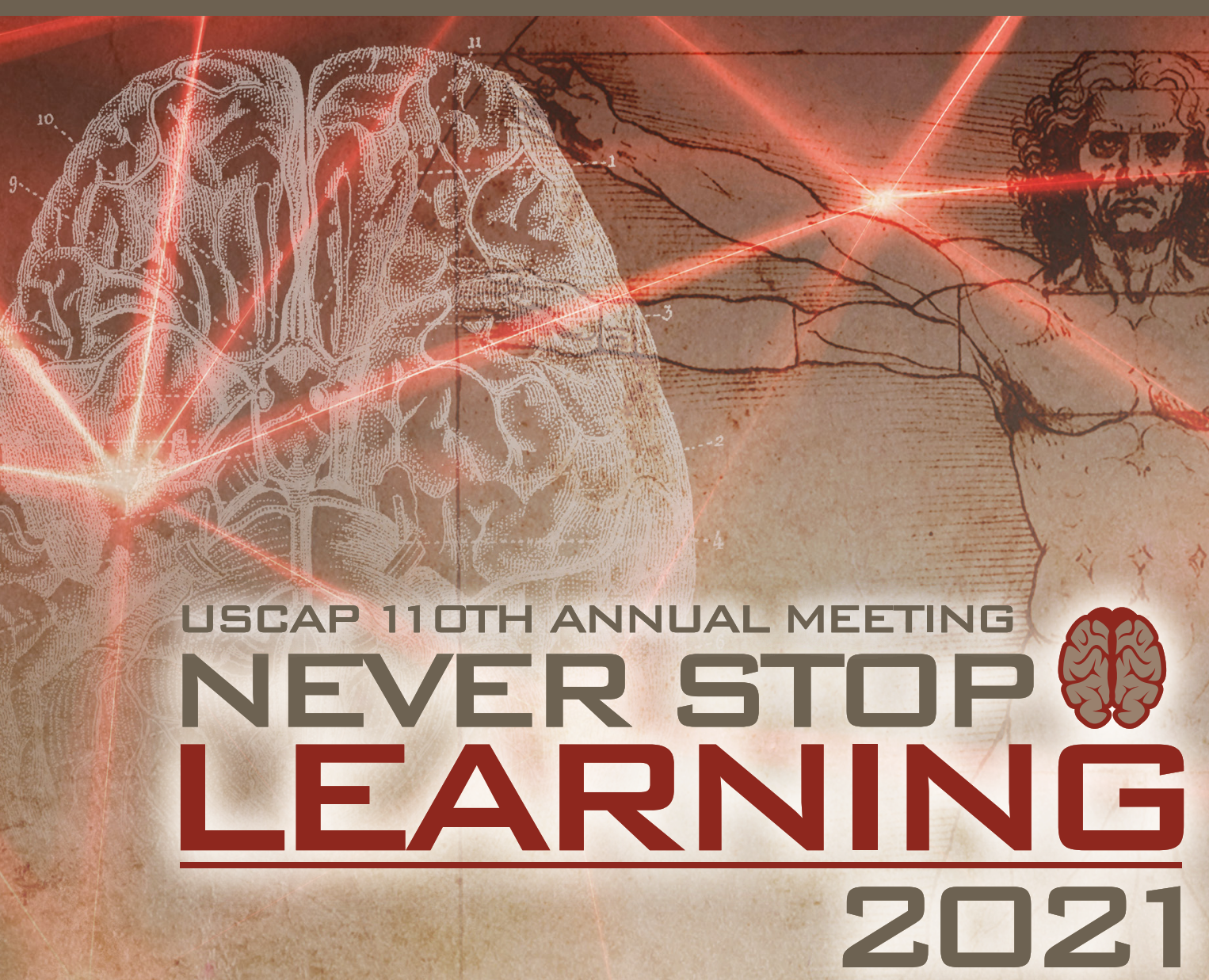

MARCH 13-18, 2021 VIRTUAL AND INTERACTIVE 


\section{EDUCATION COMMITTEE}

Jason L. Hornick

Chair

Rhonda K. Yantiss, Chair

Abstract Review Board and Assignment Committee

Kristin C. Jensen

Chair, CME Subcommittee
Laura C. Collins

Interactive Microscopy Subcommittee

Raja R. Seethala

Short Course Coordinator

Ilan Weinreb

Subcommittee for Unique Live Course Offerings

\author{
David B. Kaminsky \\ (Ex-Officio) \\ Zubair W. Baloch \\ Daniel J. Brat \\ Sarah M. Dry \\ William C. Faquin \\ Yuri Fedoriw \\ Karen Fritchie \\ Jennifer B. Gordetsky \\ Melinda Lerwill \\ Anna Marie Mulligan
}

\author{
Liron Pantanowitz \\ David Papke, \\ Pathologist-in-Training \\ Carlos Parra-Herran \\ Rajiv M. Patel \\ Deepa T. Patil \\ Charles Matthew Quick \\ Lynette M. Sholl \\ Olga K. Weinberg \\ Maria Westerhoff \\ Nicholas A. Zoumberos, \\ Pathologist-in-Training
}

\section{ABSTRACT REVIEW BOARD}

\author{
Benjamin Adam \\ Rouba Ali-Fehmi \\ Daniela Allende \\ Ghassan Allo \\ Isabel Alvarado-Cabrero \\ Catalina Amador \\ Tatjana Antic \\ Roberto Barrios \\ Rohit Bhargava \\ Luiz Blanco \\ Jennifer Boland \\ Alain Borczuk \\ Elena Brachtel \\ Marilyn Bui \\ Eric Burks \\ Shelley Caltharp \\ Wenqing (Wendy) Cao \\ Barbara Centeno \\ Joanna Chan \\ Jennifer Chapman \\ Yunn-Yi Chen \\ Hui Chen \\ Wei Chen \\ Sarah Chiang \\ Nicole Cipriani \\ Beth Clark \\ Alejandro Contreras \\ Claudiu Cotta \\ Jennifer Cotter \\ Sonika Dahiya \\ Farbod Darvishian \\ Jessica Davis \\ Heather Dawson \\ Elizabeth Demicco \\ Katie Dennis \\ Anand Dighe \\ Suzanne Dintzis \\ Michelle Downes
}

Charles Eberhart Andrew Evans

Julie Fanburg-Smith Michael Feely

Dennis Firchau

Gregory Fishbein

Andrew Folpe

Larissa Furtado

Billie Fyfe-Kirschner

Giovanna Giannico

Christopher Giffith

Anthony Gill

Paula Ginter

Tamar Giorgadze

Purva Gopal

Abha Goyal

Rondell Graham

Alejandro Gru

Nilesh Gupta

Mamta Gupta

Gillian Hale

Suntrea Hammer

Malini Harigopal

Douglas Hartman

Kammi Henriksen

John Higgins

Mai Hoang

Aaron Huber

Doina Ivan

Wei Jiang

Vickie Jo

Dan Jones

Kirk Jones

Neerja Kambham

Dipti Karamchandani

Nora Katabi

Darcy Kerr

Francesca Khani
Joseph Khoury

Rebecca King

Veronica Klepeis

Christian Kunder

Steven Lagana

Keith Lai

Michael Lee

Cheng-Han Lee

Madelyn Lew

Faqian Li

Ying Li

Haiyan Liu

Xiuli Liu

Lesley Lomo

Tamara Lotan

Sebastian Lucas

Anthony Magliocco

Kruti Maniar

Brock Martin

Emily Mason

David McClintock Anne Mills

Richard Mitchell

Neda Moatamed

Sara Monaco

Atis Muehlenbachs Bita Naini

Dianna Ng

Tony $\mathrm{Ng}$

Michiya Nishino

Scott Owens

Jacqueline Parai

Avani Pendse

Peter Pytel

Stephen Raab

Stanley Radio

Emad Rakha

Robyn Reed
Michelle Reid

Natasha Rekhtman

Jordan Reynolds

Andres Roma

Lisa Rooper

Avi Rosenberg

Esther (Diana) Rossi

Souzan Sanati

Gabriel Sica

Alexa Siddon

Deepika Sirohi

Kalliopi Siziopikou

Maxwell Smith

Adrian Suarez

Sara Szabo

Julie Teruya-Feldstein

Khin Thway

Rashmi Tondon

Jose Torrealba

Gary Tozbikian

Andrew Turk

Evi Vakiani

Christopher VandenBussche

Paul VanderLaan

Hannah Wen

Sara Wobker

Kristy Wolniak

Shaofeng Yan

Huihui Ye

Yunshin Yeh

Anjana Yeldandi

Gloria Young

Lei Zhao

Minghao Zhong

Yaolin Zhou

Hongfa Zhu

To cite abstracts in this publication, please use the following format: Author A, Author B, Author C, et al. Abstract title (abs\#). In "File Title." Laboratory Investigation 2021; 101 (suppl 1): page\# 


\section{Polymorphous Adenocarcinoma: Clinicopathologic Appraisal of 55 Cases from A Single} Institution

Emad Ababneh ${ }^{1}$, Bryan Hair², Brian Burkey², Shlomo Koyfman², Mobeen Rahman³, Deborah Chute², Christopher Griffith ${ }^{1}$

${ }^{1}$ Cleveland Clinic Foundation, Cleveland, $\mathrm{OH},{ }^{2} \mathrm{Cleveland}$ Clinic, Cleveland, $\mathrm{OH},{ }^{3} \mathrm{Cleveland}$ Clinic Pathology and Laboratory Medicine, Cleveland, $\mathrm{OH}$

Disclosures: Emad Ababneh: None; Brian Burkey: None; Mobeen Rahman: None; Deborah Chute: None; Christopher Griffith: None

Background: Polymorphous adenocarcinoma (PAC) is a rare salivary gland malignancy characterized by diverse histologic patterns. This study aims to analyze pathologic and clinical findings to refine diagnostic and prognostic findings of significance.

Design: All cases with a diagnosis of PAC, cribriform adenocarcinoma of minor salivary gland or polymorphous low-grade adenocarcinoma from 2000-2019 are included. Clinical and pathologic features were reviewed.

Results: 55 cases are reviewed. The clinical and pathologic features are presented in table 1. 29 cases had cribriform pattern representing $>30 \%$ of tumor architecture. One tumor demonstrated 21 mitotic figures per 10 HPF, focal necrosis, and pleomorphism combined. Immunohistochemistry was available for review on a subset and showed nearly all tumors to be diffusely positive for p63, S100, SOX10, and negative for P40. Specific myoepithelial markers were variable but commonly demonstrated focal to patchy reactivity (see table 1). Clinical and follow-up data is available on 28 cases (median 203 weeks, range 1.6-1170). 8 cases presented with advanced local disease (pT4) and 6 cases with lymph node metastasis. Patients were treated with surgery alone $(n=20)$ or with postop radiation $(n=7)$. One patient received chemoradiation without surgery. Distant metastases are not present. Margins were positive in 7 cases and close $(<1 \mathrm{~mm})$ in 6 . Locoregional recurrence occurred in 6 (median time to recurrence is 186.7 weeks, range 24.5-917). Among recurrent cases, 4 patients had locally advanced primary disease and 3 had lymph node metastasis at presentation. 3 cases had positive/close margin status and $5 / 6$ showed PNI. Cribriform growth was prominent ( $>30 \%)$ in only 2 cases. 3 recurrent cases had increased mitotic activity (4-6 mitosis/10 HPF) and 1 had nuclear pleomorphism.

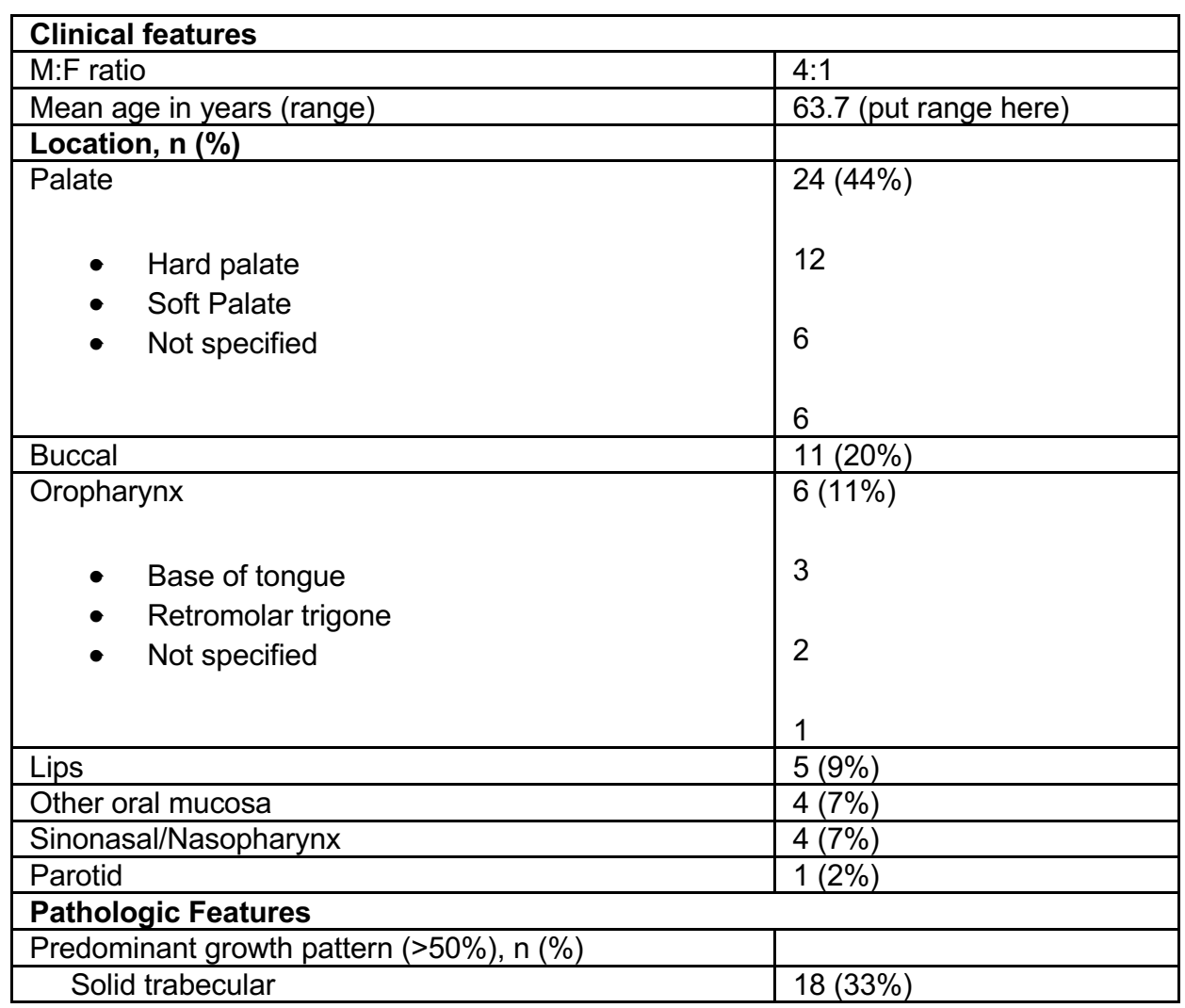




\begin{tabular}{|l|l|} 
Cribriform & $10(18 \%)$ \\
\hline Tubular & $5(9 \%)$ \\
\hline Papillary & $4(7 \%)$ \\
\hline No predominant pattern & $18(33 \%)$ \\
\hline Apocrine change, $\mathrm{n}(\%)$ & $14(25 \%)$ \\
\hline Oncocytic change, $\mathrm{n}(\%)$ & $2(4 \%)$ \\
\hline Significant pleomorphism & $2(4 \%)$ \\
\hline Necrosis & $2(4 \%)$ \\
\hline$>3$ Mitoses/10 HPF, $\mathrm{n}(\%)$ & $11(20 \%)$ \\
\hline Perineural Invasion, $\mathrm{n}(\%)$ & $23(42 \%)$ \\
\hline Lymphovascular invasion, $\mathrm{n}(\%)$ & $11(20 \%)$ \\
\hline Immunohistochemistry, $\mathbf{n}(\%)$ & \\
\hline P63 & $38 / 39(97 \%)$ \\
\hline S100 & $36 / 37(97 \%)$ \\
\hline SOX10 & $23 / 23(100 \%)$ \\
\hline P40 & $0 / 18(0 \%)$ \\
\hline SMA & $13 / 35(37 \%)$ \\
\hline SMMS & $27 / 30(90 \%)$ \\
\hline AR & $4(29 \%)$ \\
\hline
\end{tabular}

Conclusions: Polymorphous adenocarcinoma is generally a low-grade neoplasm with diverse histologic patterns. The immune profile consistently shows diffuse positivity for P63, S100, and SOX10. Focal myoepithelial marker expression is common. Apocrine/oncocytic change can be seen in some cases. Histologic and cytologic findings are not strong predictors of local recurrence, but half of the patients presenting with advanced pT4 disease or lymph node metastases at presentation experienced subsequent recurrence.

\section{Clinicopathologic Characteristics and Prognostic Factors of Primary and Recurrent Pleomorphic Adenoma: A Single Institution Retrospective Study of 251 Cases Bayan Alzumaili' ${ }^{1}$, Bin $\mathrm{Xu}^{2}$, Maelle Saliba², Anjanie Khimraj², Ronald Ghossein ${ }^{2}$, Nora Katabi ${ }^{2}$ ${ }^{1}$ Mount Sinai Health System, New York, NY, ${ }^{2}$ Memorial Sloan Kettering Cancer Center, New York, NY}

Disclosures:Bayan Alzumaili: None; Maelle Saliba: None; Anjanie Khimraj: None; Ronald Ghossein: None; Nora Katabi: None

Background: Pleomorphic adenoma (PA), characterized by a variable morphology, is the most common salivary gland neoplasm. Despite its benign classification, it has the capacity to locally recur, and may prompt further treatment with radiotherapy (RT). To further investigate the prognostic features of PA, we conducted a detailed clinicopathologic study on a large cohort of this tumor.

Design: We analyzed 251 PAs from 239 patients, including 186 primary and 65 recurrent tumors. The patient age, site and size, histopathologic features, and clinical management were documented, and recurrence free survival (RFS) was calculated.

Results: Follow up (median: 5.5 years, range: 0.5-37.0 years) was available on 186 primary PAs, of which 8 (4\%) developed local recurrences (7 were PAs, and 1 had malignant transformation). The clinicopathologic features of the primary and recurrent PAS are demonstrated in table 1. Patient age, tumor site, increased mitoses, high cellularity, and nodular borders correlated with RFS on univariate analysis using the log rank test. Only patient age and high cellularity were statistically significant on multivariate analysis using the Cox proportional hazard model $(p<0.05)$.

The median time for first recurrence (R1) was 7.5 years (range: 0.5-33.7 years). Out of 61 patients with R1, 31 $(51 \%)$ had subsequent recurrences (R2-R5), with the following median time: R1-R2=5.6 years (range: 0.8-39.8 years, 30 patients), R2-R3=7.6 years (range: $1.3-16.3$ years, 8 patients), R3-R4=7.0 years (range: 1.0-13.0 years, 2 patients) and R4-R5=16.2 years (1 patient). Of the 61 patients with R1, $22(36 \%)$ were treated with RT. Of interest, 9/39 (23\%) patients who did not receive RT had tumor progression versus only $2 / 22$ (9\%) patients who did receive RT. 
Table-1: Clinicopathologic features of PAs.

\begin{tabular}{|c|c|c|c|}
\hline \multicolumn{2}{|l|}{$\begin{array}{l}\text { Clinicopathologic features } \\
\text { of primary PAs }\end{array}$} & \multicolumn{2}{|l|}{$\begin{array}{l}\text { Clinicopathologic features } \\
\text { of recurrent PAs }\end{array}$} \\
\hline Median age (range) & 50 years $(12-86)$ & Median age (range) & 30 years $(12-74)$ \\
\hline Sex & & Sex & \\
\hline Male & $32 \%(60 / 186)$ & Male & $34 \%(21 / 61)$ \\
\hline Female & $68 \%(126 / 186)$ & Female & $66 \%(40 / 61)$ \\
\hline Site & & Site of primary & \\
\hline Parotid & $89 \%(166 / 186)$ & Parotid & $87 \%(53 / 61)$ \\
\hline Submandibular & $7 \%(13 / 186)$ & Submandibular & $10 \%(6 / 61)$ \\
\hline Minor salivary gland & $4 \%(7 / 186)$ & Minor salivary gland & $3 \%(2 / 61)$ \\
\hline $\begin{array}{l}\text { Median size of primary } \\
\text { nodule (range), } 185 \\
\text { cases }\end{array}$ & $2.0 \mathrm{~cm}(0.6-6.2 \mathrm{~cm})$ & $\begin{array}{l}\text { Median size of largest R1 } \\
\text { nodule (range), } 36 \text { cases }\end{array}$ & $1.3 \mathrm{~cm}(0.1-5.0 \mathrm{~cm})$ \\
\hline Tumor cellularity & & Tumor cellularity (R1) & \\
\hline$\leq 50 \%$ & $59 \%(109 / 186)$ & $\leq 20 \%$ & $71 \%(27 / 38)$ \\
\hline$>50 \%$ & $41 \%(77 / 186)$ & $>20 \%$ & $29 \%(11 / 38)$ \\
\hline Number of pseudopods & & Number of nodules (R1) & \\
\hline 0 & $53 \%(99 / 186)$ & $\leq 10$ & $47 \%(18 / 38)$ \\
\hline $1-10$ & $42 \%(78 / 186)$ & $>10$ & $53 \%(20 / 38)$ \\
\hline$>10$ & $5 \%(9 / 186)$ & & \\
\hline Mitosis & & Mitosis (R1) & \\
\hline Absent & $61 \%(113 / 186)$ & Absent & $76 \%(29 / 38)$ \\
\hline Present & $39 \%(73 / 186)$ & Present & $24 \%(9 / 38)$ \\
\hline Nodular borders & $96 \%(179 / 186)$ & Margins (R1) & \\
\hline Irregular tumor periphery & $31 \%(57 / 182)$ & Positive & $42 \%(15 / 36)$ \\
\hline Tumor encapsulation & & Negative & $58 \%(21 / 36)$ \\
\hline Complete & $64 \%(114 / 179)$ & & \\
\hline Partial or no capsule & $36 \%(65 / 179)$ & & \\
\hline Lymphovascular invasion & $<1 \%(1 / 186)$ & & \\
\hline Perineural invasion & $<1 \%(1 / 186)$ & & \\
\hline Tumor necrosis & $0 \%(0 / 186)$ & & \\
\hline Margins & & & \\
\hline Positive & $14 \%(25 / 185)$ & & \\
\hline Negative & $86 \%(160 / 185)$ & & \\
\hline
\end{tabular}

Conclusions: In this study, the recurrence rate of primarily resected PA was $4 \%$ and the risk of malignant transformation was $<1 \%$. Patient age, tumor site, increased mitoses, high cellularity, and nodular borders correlated with RFS on univariate analysis, but only patient age and high cellularity were independent prognostic factors on multivariate analysis.

\section{Assessment of Tumor-Infiltrating Lymphocytes in Head and Neck Squamous Cell Carcinoma on H\&E-stained Slides: Visual Versus Computer-Assisted Evaluation, Association with Clinicopathological Features and Survival Javier Baena-Del Valle ${ }^{1}$, Mauricio Palau ${ }^{1}$, Ana Margarita Baldión-Elorza ${ }^{1}$, Catalina Buritica-Cifuentes ${ }^{1}$, Patricia Bernal-Trujillo ${ }^{1}$, Gonzalo Ucros-Rodriguez ${ }^{1}$, Alvaro Munoz Perez ${ }^{1}$, Aylen Vanessa Ospino- Serrano ${ }^{1}$, Javier Segovia-Gómez ${ }^{1}$, Alberto Escallón-Cubillos ${ }^{1}$, Paula Andrea Rodriguez - Urrego ${ }^{2}$ ${ }^{1}$ Fundacion Santa Fe de Bogota University Hospital, Bogota, Colombia, ${ }^{2}$ University Hospital Fundacion Santa Fe de Bogota, Esmeraldal, Colombia}

Disclosures: Javier Baena-Del Valle: None; Mauricio Palau: None; Ana Margarita Baldión-Elorza: None; Catalina Buritica-Cifuentes: None; Patricia Bernal-Trujillo: None; Gonzalo Ucros-Rodriguez: None; Alvaro Munoz Perez: None; Aylen Vanessa Ospino-Serrano: None; Javier Segovia-Gómez: None; Alberto Escallón-Cubillos: None; Paula Andrea Rodriguez - Urrego: None

Background: Head and neck squamous cell carcinoma (HNSCC) is one of the top ten most frequent and lethal cancers worldwide. Despite conventional treatment, local recurrence and metastasis are still frequent, requiring 
newer and effective therapeutic strategies. Recently, the tumor microenvironment has emerged and important predictor of biological behavior and response to treatment in tumors, specially the density of tumor infiltrating lymphocytes (TILs). In HNSCC more scientific evidence of the impact of TILs in prognosis is needed. Moreover, there are currently no standards for quantification (cut-offs, visual versus digital assessment). The aim of this study was to quantify TILs in HNSCC H\&E sections and its association with clinicopathological features and survival. Also, to compare visual versus computer-assisted scoring.

Design: 40 formalin-fixed and paraffin-embedded samples from HNSCC patients from the Colombian cohort of the InterCHANGE study (IARC) were included. Mean follow up time was 36 months from diagnosis. Low-density TMAs were constructed, and the slides were stained with H\&E. Digital quantification of TILs was performed by separating the intratumor stroma using an object classifier, followed by a cell classification based on nuclear/cytoplasmic ratio and smoothed features (Fig. 1). Visual quantification of the density of TILs in the intratumor stroma was performed by 4 pathologists.

Results: Similar TILs density was observed among the anatomic subsites. Higher TILs density ( $\geq 30 \%)$ was associated with improved overall survival $(p=0.048)$, clinical stage IV (only significant when compared to stage II, $p=0.043$ ) and positive surgical margins, the latter only in oral cavity tumors $(p=0.020)$ (Fig. 2). there was no significant association with smoking status, alcohol, p16 status, gender, bone invasion, lymph node metastasis, tumor size and depth of invasion. Digital versus visual assessment showed a substantial agreement (quadratic weighted kappa=0.7), and an equally significant inter-observer agreement between pathologists (inter-observer agreement coefficient $=0.64$ ).

A

Figure $1-637$
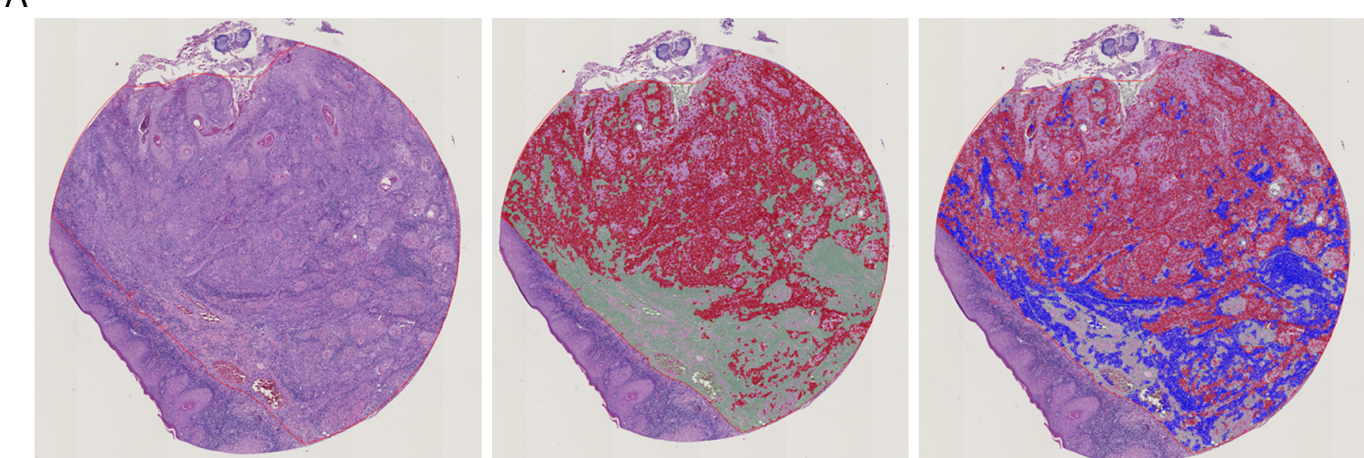

An example of a digital tumor and stroma segmentation using a trained-object classifier, followed by detection of immune cells

$\mathrm{B}$

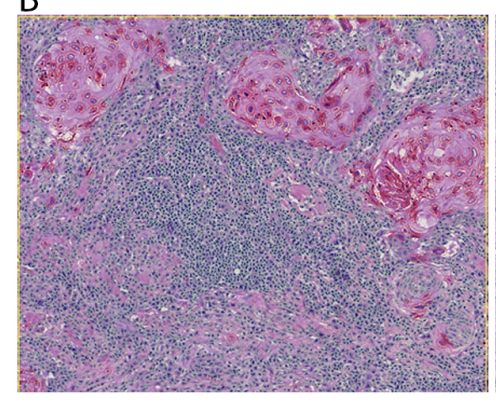

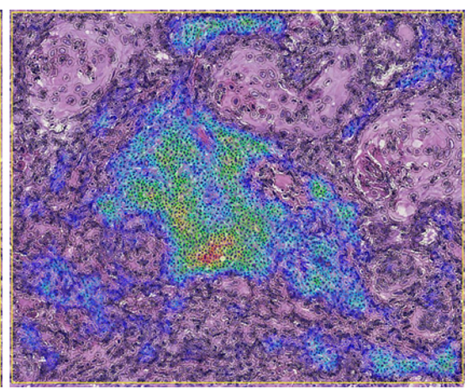

Nuclear-cytoplasmic ratio, supplemented with smoothed object features at $25 \mu \mathrm{m}$ radius were used to accurately classify cells as immune (TILs). 
Figure 2 - 637

A Percentage of Intratumor Stroma Occupied by TILs According to Tumor Site

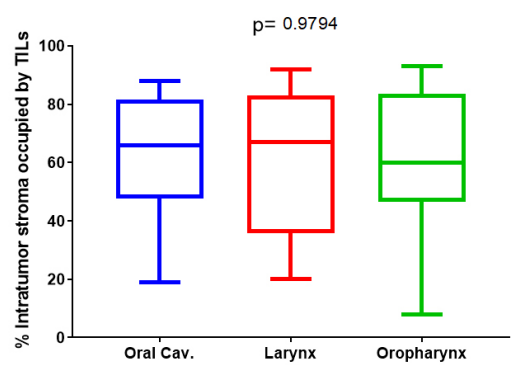

C

Percentage of Intratumor Stroma Occupied by TILs According to Margin Status in Oral Cavity Tumors

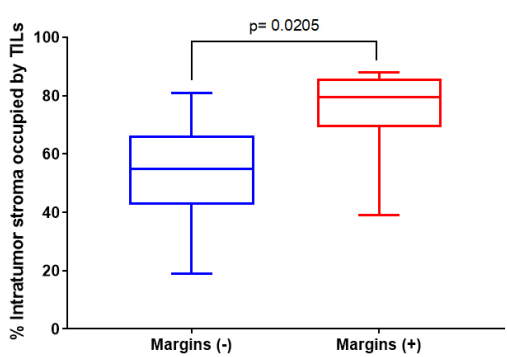

B

Percentage of Intratumor Stroma Occupied by TILs According to

TNM Staging (8th Ed) $p=0.3238$

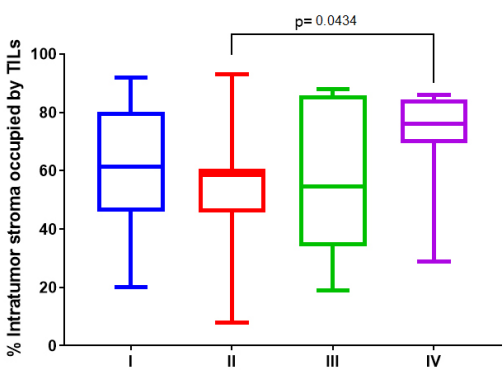

D

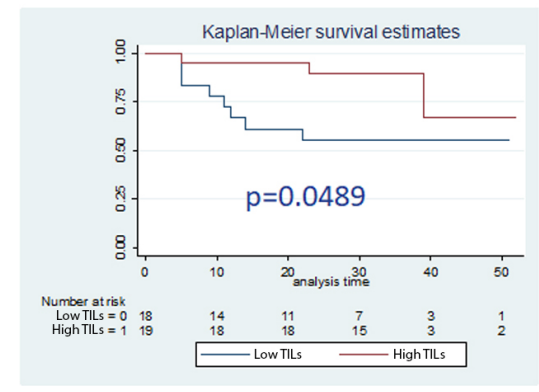

Conclusions: We show that density of TILs, regardless of their type, may be associated with survival and important clinicopathological parameters. We also show that visual scoring has significant agreement with digital ones and could be used with an acceptable level of agreement. However, in cases when the scores are close to a limit of a category, digital analysis can be useful to proper classify the density of the infiltrate.

\section{Quantitative Tissue Architecture Features are Statistically Similar Between Human- Annotated and Al-Generated Tumor Label Maps in Early-Stage Oral Cavity Cancer Dhadma Balachandran ${ }^{1}$, Margaret Brandwein-Weber ${ }^{2}$, Jonathan Folmsbee ${ }^{1}$, Scott Doyle ${ }^{1}$ \\ ${ }^{1}$ University at Buffalo, SUNY, Buffalo, NY, ${ }^{2}$ Icahn School of Medicine at Mount Sinai, New York, NY}

Disclosures: Dhadma Balachandran: None; Margaret Brandwein-Weber: None; Jonathan Folmsbee: None; Scott Doyle: None

Background: Patients with early-stage (Stage I and II) Oral Cavity Cancer (OCC) experience a high degree of loco-regional recurrence (LRR) with $25-37 \%$ of patients requiring additional follow-up. The Histological Risk Model (HRM) is a clinically validated model which predicts LRR. "Worst Pattern of Invasion" (WPOI) is a predictive histologic features of the HRM which classifies tumor invasion phenotypes based on dispersion of "satellite" tumors (ST) that are convincingly separate from the main tumor (MT). Our goal is to build a Quantitative Risk Model (QRM) as an extension of HRM to automate the prediction process by extracting quantitative features from computer generated tissue maps.

To accomplish this, we have built an automated Artificial Intelligence (AI) tissue classification pipeline. In this work we are concerned with the accuracy of Al-generated maps. Specifically, we investigate: (1) How can we automatically designate tumor regions as ST vs. MT? (2) Are the Al tissue maps sufficiently accurate to generate WPOI features comparable with human annotated maps?

Our hypothesis is that segmentation accuracy should be determined by similarity of extracted features rather than comparison against a "perfect" decontextualized segmentation standard, as these quantitative features are the ones that eventually influence the QRM 
Design: Pathologists trained in HRM selected 49 region of interest (ROI) from 45 digitally scanned WSI and handannotated tumor and satellite label maps (Figure 1). 80 architectural features were extracted from each of the 49 ROI. Next, all hand-labeled carcinoma regions were relabeled into ST and MT using a rigid heuristic. Our heuristic considered objects with the largest contiguous area to be MT and the remaining tissues regions as ST. This same heuristic was applied to Al-generated maps using a semantic segmentation classifier (previously developed). Our three experimental groups (Figure 2) are: (A) hand-annotated ground truth maps with manual MT and ST labels;

(B) tumor maps from (A) but with heuristically-assigned MT and ST; and (C) Al tumor maps with the same MT and ST heuristic. The extracted feature sets from group (B) and (C) are then statistically compared using a paired t-test to determine whether the relabeling process has a statistically significant effect on the value of each feature (Table 1).

Results: Significant similarity was observed in $87.5 \%$ (10 features) of the 80 distinct features extracted from Al tumor maps versus corresponding ground truth maps (with heuristic MT and ST labels). This supports the feasibility of using computer generated tissue maps for QRM development.

\begin{tabular}{|c|c|c|c|c|c|}
\hline & Convex hull & ST to MT distance & Triangle area & Triangle length & Satellite wave \\
\hline Minimum & 0.136082017 & 0.189724392 & 0.02721031 & $7.61 \mathrm{E}-05$ & 0.277153799 \\
\hline Maximum & 0.448900218 & 0.92756103 & 0.695346046 & 0.13132854 & 0.895327919 \\
\hline Mean & 0.608196093 & 0.808382972 & 0.065944165 & 0.00474962 & 0.846375641 \\
\hline Variance & 0.937660944 & 0.670336541 & 0.619755548 & 0.991045196 & 0.852440299 \\
\hline $\begin{array}{l}\text { Standard } \\
\text { deviation }\end{array}$ & 0.663257105 & 0.797411371 & 0.928291606 & 0.292086919 & 0.997901475 \\
\hline Skewness & 0.609924593 & 0.366537058 & 0.000445391 & 0.332117902 & 0.289958244 \\
\hline Kurtosis & 0.805187535 & 0.682942777 & 0.00292673 & 0.402199017 & 0.252431722 \\
\hline Moment 5 & 0.382091129 & 0.057338062 & 0.981465304 & 0.734826272 & 0.047147796 \\
\hline Moment 6 & 0.353230071 & 0.742577573 & 0.848096314 & 0.546017349 & 0.662169468 \\
\hline Moment 7 & 0.338943154 & 0.100673604 & 0.740232028 & 0.420037571 & 0.088083721 \\
\hline Moment 8 & 0.331504642 & 0.547065708 & 0.65154628 & 0.346985681 & 0.508340692 \\
\hline Moment 9 & 0.327480543 & 0.162948147 & 0.57931449 & 0.304618587 & 0.140603441 \\
\hline Moment 10 & 0.325251603 & 0.440427366 & 0.521656843 & 0.279731852 & 0.42595401 \\
\hline Moment 11 & 0.323998299 & 0.219500325 & 0.476470125 & 0.264899054 & 0.189837288 \\
\hline $\begin{array}{l}\text { Geometric } \\
\text { mean }\end{array}$ & 0.332877078 & 0.499243361 & 0.00080784 & $2.59 \mathrm{E}-05$ & 0.767287065 \\
\hline Harmonic mean & 0.204526447 & 0.236708427 & 0.000739334 & 2.74E-07 & 0.631842412 \\
\hline
\end{tabular}


Figure $1-638$

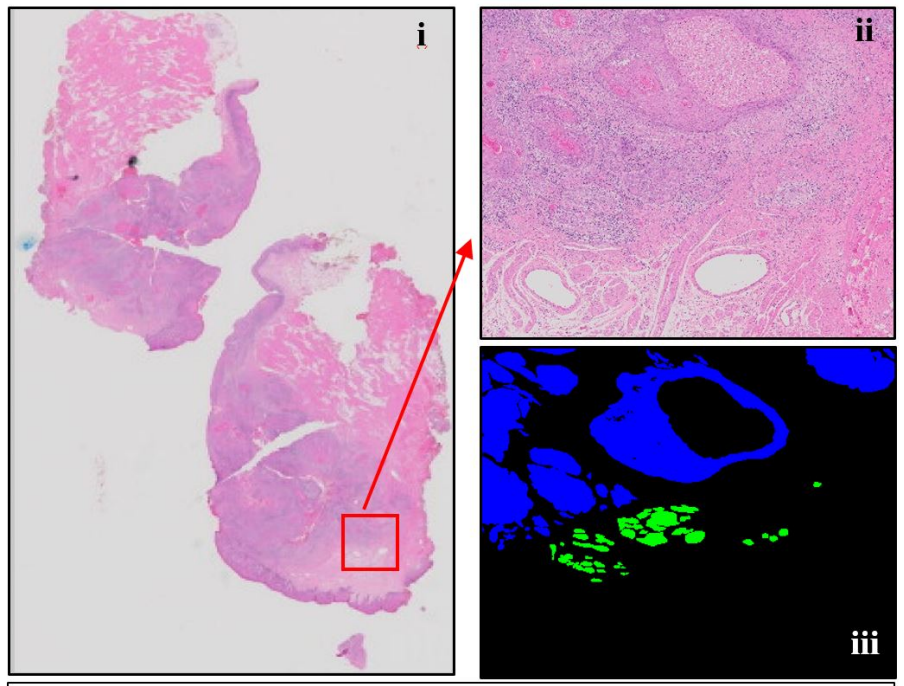

Figure 1 (i) Shows an example of whole slide image, (ii) is the extracted region of interest from whole slide image, and (iii) is the hand-annotated tumor maps.
Figure 2 - 638

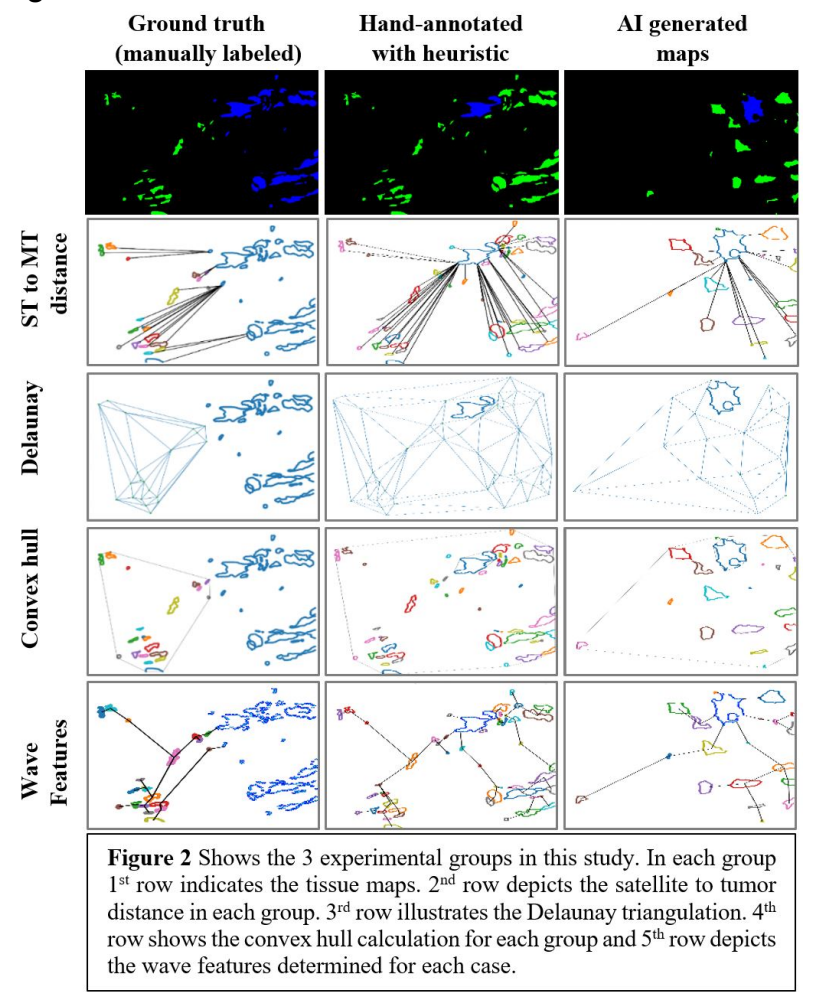

Conclusions: Our experimental results support our hypothesis that qualitative differences in tumor-satellite maps has minimal influence on the architectural feature values extracted after accounting for the heuristic ST vs. MT labeling. This work is crucial to developing a reliable QRM for early stage OCC patients.

\section{Acinic Cell Carcinoma: NR4A3 Fluorescence In Situ Hybridization Analysis in 40 Cytologic and Surgically Resected Specimens \\ Caroline Bsirini ${ }^{1}$, Bin Zhang ${ }^{2}$, LiQiong Li ${ }^{2}$, Ellen Giampoli², Kelly Magliocca ${ }^{3}$, Michelle Reid ${ }^{1}$, Vaidehi \\ Avadhani ${ }^{1}$, Quying Shi ${ }^{3}$, Zhongren Zhou ${ }^{4}$ \\ ${ }^{1}$ Emory University Hospital, Atlanta, GA, ${ }^{2}$ University of Rochester Medical Center, Rochester, NY, ${ }^{3}$ Emory University, Atlanta, GA, ${ }^{4}$ Rutgers University, New Brunswick, NJ}

Disclosures: Caroline Bsirini: None; Bin Zhang: None; LiQiong Li: None; Ellen Giampoli: None; Kelly Magliocca: None; Michelle Reid: None; Vaidehi Avadhani: None; Qiuying Shi: None; Zhongren Zhou: None

Background: Because of its highly variable histology including potential high-grade transformation, acinic cell carcinoma (ACC) may pose a diagnostic challenge particularly on small biopsies and fine needle aspiration (FNA). A novel rearrangement $t(4 ; 9)(q 13 ; q 31)$ leading to upregulation of nuclear receptor subfamily 4 group $A$ member 3 (NR4A3) has been described in ACC and is potentially detectable by FISH. Breakpoint may/may not immediately adjacent to the NR4A3 gene and escape detection by FISH.

Design: Using ZytoLight ${ }^{\circledR}$ SPEC NR4A3 Dual Color Break Apart Probe (Zytovision, Germany) FISH was performed on ACCs from three large academic institutions. NR4A3 rearrangement was defined as positive signal patterns in $15 \%$ of tissue interphase nuclei. Clinicopathologic features of FISH positive (+) and FISH negative (-) ACC were compared.

Results: 40 ACCs including 32 resections and 8 FNAs (including 5 paired FNA/resections) were analyzed. 5 nonACC salivary gland tumors and 2 sialadenitis cases were used as controls. 4(10\%) ACCs failed FISH testing. FISH was (+) in 18 ACCs (sensitivity 58\%, 18/31) with 100\% concordance between five matched resection/FNAs (three were $\mathrm{FISH}(+)$ and two were FISH (-)). FISH was (-) in all non-ACCs (specificity: 100\%, 0/7). No specific 
morphologic differences between $4 \mathrm{FISH}(+)$ vs $4 \mathrm{FISH}(-)$ ACCs, in that both groups had a wide morphologic spectrum (architecture, presence and quantity of zymogen, microcysts, tumor associated lymphoid proliferation and high-grade transformation).

Figure 1 - 639

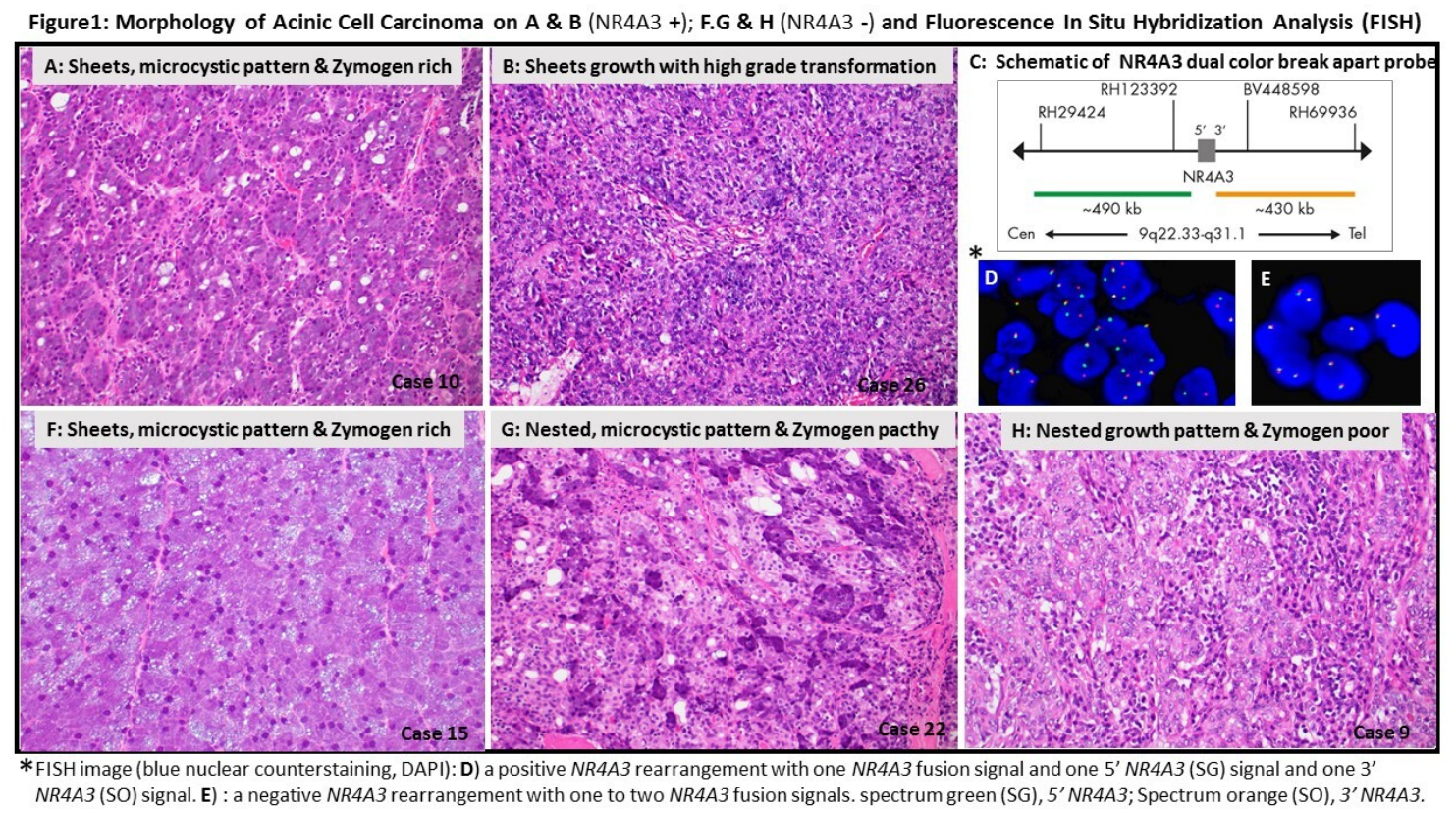

Conclusions: NR4A3 FISH has a sensitivity of $58 \%$ and specificity of $100 \%$ in detecting ACC which suggests that NR4A3 rearrangement-driven upregulation is a recurrent, specific oncogenic event in ACC, consistent with prior results. $100 \%$ concordance between matched resections/FNAs validates its potential utility on cytology samples. FISH (+) ACC had no specific morphologic correlates.

\section{BRAF VE1 Immunoreactivity Distinguishes Ameloblastoma from Keratocystic Odontogenic Tumor and Dentigerous Cyst, and Correlates with BRAF V600E Mutation Status \\ Luvy Delfin Mendez ${ }^{1}$, Nicholas Wolsefer ${ }^{2}$, Sylvia Asa ${ }^{1}$, Marta Couce ${ }^{3}$, Jay Wasman ${ }^{3}$, Jennifer Yoest ${ }^{1}$, Ivan Stojanov ${ }^{2}$ \\ ${ }^{1}$ Case Western Reserve University/University Hospitals Cleveland Medical Center, Cleveland, $\mathrm{OH},{ }^{2}$ Case Western Reserve University, Cleveland, $\mathrm{OH},{ }^{3}$ University Hospitals Cleveland Medical Center, Cleveland, $\mathrm{OH}$}

Disclosures: Luvy Delfin Mendez: None; Nicholas Wolsefer: None; Sylvia Asa: Advisory Board Member, Leica Biosystems; Marta Couce: None; Jay Wasman: None; Jennifer Yoest: None; Ivan Stojanov: None

Background: Ameloblastoma, keratocystic odontogenic tumor (KCOT) and dentigerous cyst (DC) are frequent diagnostic considerations in the evaluation of cystic lesions from the mandible or maxilla. These conditions pursue distinct clinical courses and are treated differently, yet their diagnosis on biopsy can be challenging when tissue is limited and there are no known helpful immunohistochemical adjuncts. Ameloblastomas are known to harbor $B R A F^{\mathrm{V} 600 \mathrm{E}}$ mutation in a majority of cases and these mutations have been shown to be identified using the VE1 antibody by immunohistochemistry, but the diagnostic utility of BRAF VE1 immunohistochemistry in this setting has not been previously addressed. The aim of this study was to analyze BRAF VE1 immunoreactivity in ameloblastoma, KCOT and DC and to correlate BRAF VE1 immunoreactivity with $B R A F^{\mathrm{V} 600 \mathrm{E}}$ mutation status in ameloblastoma. 
Design: 30 ameloblastomas, $30 \mathrm{KCOTs}$ and $30 \mathrm{DCs}$ not subjected to decalcification were retrieved from institutional archives. BRAF VE1 immunohistochemistry was performed on all 90 cases and DNA was extracted from FFPE tissue of the 30 ameloblastomas and subjected to allele-specific polymerase chain reaction (AS-PCR) to determine $B R A F^{\mathrm{V} 600 \mathrm{E}}$ mutation status. Immunohistochemistry interpretation was blinded to AS-PCR results.

Results: Ameloblastomas occurred in 16 male and 14 female patients with a median age of 65 (range: 16-85) years. 23 ameloblastomas were located in the mandible and 7 in the maxilla. BRAF VE1 staining was positive in $19 / 23(83 \%)$ mandibular ameloblastomas and negative in all maxillary ameloblastomas (0/7), KCOTs (0/30) and DCs $(0 / 30)$. Positive cases included one with diffuse nuclear staining and another with weak/equivocal staining. ASPCR demonstrated BRAF ${ }^{\mathrm{V} 600 \mathrm{E}}$ mutation in 19/30 ameloblastomas, with BRAF VE1 immunohistochemistry demonstrating $100 \%$ specificity and sensitivity.

Conclusions: BRAF VE1 immunoreactivity is specific for ameloblastoma in the differential diagnosis of KCOT and DC. BRAF VE1 is positive in $83 \%$ of mandibular ameloblastomas and negative in all maxillary ameloblastomas, KCOTs and DCs tested, and is valuable in the workup of diagnostically challenging cystic mandibular tumors but is of limited utility in the maxilla. BRAF VE1 reactivity, moreover, correlates with $B R A F^{\mathrm{V} 600 \mathrm{E}}$ mutation status and known patterns of $B R A F^{\mathrm{V} 600 \mathrm{E}}$ mutation in ameloblastoma, and represents a rational screening tool for patient identification for targeted therapeutic approaches.

\section{Evaluation of PD-L1 Immunohistochemical Expression in the Head and Neck Squamous Cell Carcinoma and its Correlation with Various Clinicopathologic Parameters: A Study from a Tertiary Referral Center in India Aditi Dewan ${ }^{1}$, Shivani Sharma², Nitin Bhardwaj ${ }^{3}$, Ekta Jain $^{2}$, Abhishek Kumar $^{1}$, Deepika Jain ${ }^{1}$, Mallika Dixit $^{1}$, Lalit Kumar ${ }^{1}$, Rakesh Pandey ${ }^{1}$, Lata Kini' ${ }^{2}$, Sambit Mohanty ${ }^{4}$ ${ }^{1}$ Core Diagnostics, Gurugram, India, ${ }^{2}$ Core Diagnostics, Gurgaon, India, ${ }^{3}$ New Delhi, India, ${ }^{4}$ AMRI Hospital, Chandigarh, India}

Disclosures: Aditi Dewan: None; Shivani Sharma: None; Nitin Bhardwaj: None; Ekta Jain: None; Abhishek Kumar: None; Deepika Jain: None; Mallika Dixit: None; Lalit Kumar: None; Rakesh Pandey: None; Lata Kini: None; Sambit Mohanty: None

Background: PDL-L1 immunohistochemistry (IHC) 22C3 PharmDx assay was approved by FDA as a companion diagnostic test as an aid in identifying patients of head and neck squamous cell carcinoma (HNSCC) eligible for treatment with Pembrolizumab (immunotherapy). Only few isolated studies from India have evaluated the correlation between PD-L1 expression in HNSCC and various clinico-pathologic parameters.

Design: In this retrospective ongoing study we evaluated PD-L1 IHC expression using the 22C3 PharmDx assay performed on the Dako autostainer link 48 for formalin fixed paraffin embedded (FFPE) blocks from biopsy and surgical resection specimens of patients with metastatic/ recurrent HNSCC. The PD-L1 expression was evaluated using Combined positive score (CPS) with a cut-off of $\geq 1$ to define positive cases. Further, it was divided into low (1-19) and high $(\geq 20)$ expression as per the KEYNOTE 048 trial. We correlated the PD-L1 expression with the primary tumor type ( oral cavity versus pharynx versus larynx) and site of PDL-1 evaluation (primary/recurrent versus metastatic). It was also correlated with various histologic parameters including histologic grade (for all cases- Figure 1) and tumor infiltrating lymphocytes (TIL), depth of invasion (DOI), lymphovascular (LVI), perineural invasion (PNI), bone involvement, lymph node(LN) status, extranodal extension(ENE) and stage of the tumor wherever available for resection specimens.

Results: Till date we have evaluated 100 cases of HNSCC for PD-L1 expression on FFPE blocks using the 22C3 Dako antibody clone. Our cohort comprises of 81 male patients and 19 female patients with a mean age of 56.6 years. Overall PDL-1 positivity rate was $84 \%$ with $32 / 84$ (38.1\%) cases showing low PDL-1 expression and 52/84 $(61.9 \%)$ cases showing a high PDL-1 expression. The results of correlation between PD-L1 expression and various clinicopathologic parameters are illustrated in Table 1. A statistically significant correlation $(p=<0.05)$ was seen between PD-L1 expression and site of PD-L1 evaluation (primary or metastatic- $p=0.017$ ), histologic grade $(p=0.003)$ and TILs $(p=0.022)$. However, no significant correlation was seen between PD-L1 expression and the primary tumor site, DOI, LVI, PNI, nodal status, ENE, bone involvement, pT and pN stage. Additionally, no 
statistically significant correlation was found between the degree of PD-L1 expression (high versus low) and any of the above parameters.

Table1: Correlation between PD-L1 and various clinico-pathologic parameters

\begin{tabular}{|c|c|c|c|c|}
\hline \multirow{2}{*}{ Parameter } & \multirow{2}{*}{ Category } & \multicolumn{2}{|c|}{ PD-L1 status } & \multirow{2}{*}{ p-value } \\
\hline & & Positive & Negative & \\
\hline \multicolumn{2}{|c|}{ Age range (Median age) yrs } & $47-64(58.5)$ & $45-65(59.5)$ & 0.877 \\
\hline \multirow{2}{*}{ Gender } & Female & $14(16.7 \%)$ & $5(31.3 \%)$ & \multirow{2}{*}{0.173} \\
\hline & Male & $70(83.3 \%)$ & $11(68.8 \%)$ & \\
\hline \multirow{3}{*}{ Primary tumor site } & Larynx & $9(10.7 \%)$ & $4(25 \%)$ & \multirow{3}{*}{0.121} \\
\hline & Oral cavity & $53(63.1 \%)$ & $6(37.5 \%)$ & \\
\hline & Pharynx+ Paranasal & $22(26.2 \%)$ & $6(37.5 \%)$ & \\
\hline \multirow{2}{*}{ PD-L1 site of evaluation } & Metastatic & $18(21.4 \%)$ & $8(50 \%)$ & \multirow{2}{*}{0.017} \\
\hline & Primary and recurrent & $66(78.6 \%)$ & $8(50 \%)$ & \\
\hline \multirow{2}{*}{ Histologic grade } & Well and moderate & $76(90.4 \%)$ & $10(62.5 \%)$ & \multirow{2}{*}{0.003} \\
\hline & Poor & $8(9.52 \%)$ & $6(37.5 \%)$ & \\
\hline \multirow{2}{*}{$\begin{array}{l}\text { Tumor infiltrating } \\
\text { lymphocytes }\end{array}$} & Mild & $5(20 \%)$ & $3(75 \%)$ & \multirow{2}{*}{0.022} \\
\hline & Moderate and marked & $20(80 \%)$ & $1(25 \%)$ & \\
\hline \multirow{2}{*}{ Depth of invasion status } & $<=1 \mathrm{~cm}$ & $7(46.7 \%)$ & $3(50 \%)$ & \multirow{2}{*}{0.890} \\
\hline & $>1 \mathrm{~cm}$ & $8(53.3 \%)$ & $3(50 \%)$ & \\
\hline \multirow{2}{*}{$\begin{array}{l}\text { Lymphovascular } \\
\text { invasion }\end{array}$} & Absent & $7(30.4 \%)$ & $4(66.7 \%)$ & \multirow{2}{*}{0.103} \\
\hline & Present & $16(69.6 \%)$ & $2(33.3 \%)$ & \\
\hline \multirow{2}{*}{ Perineural invasion } & Absent & $5(21.7 \%)$ & $2(33.3 \%)$ & \multirow{2}{*}{0.554} \\
\hline & Present & $18(78.3 \%)$ & $4(66.7 \%)$ & \\
\hline \multirow{2}{*}{ Nodal status } & Positive & $8(36.4 \%)$ & $4(66.7 \%)$ & \multirow{2}{*}{0.184} \\
\hline & Negative & $14(63.6 \%)$ & $2(33.4 \%)$ & \\
\hline \multirow{2}{*}{ Extranodal extension } & Absent & $10(47.6 \%)$ & $5(83.3 \%)$ & \multirow{2}{*}{0.121} \\
\hline & Present & $11(52.4 \%)$ & $1(16.7 \%)$ & \\
\hline \multirow{2}{*}{ Bone involvement } & Absent & $13(50 \%)$ & $4(66.7 \%)$ & \multirow{2}{*}{0.461} \\
\hline & Present & $13(50 \%)$ & $2(33.3)$ & \\
\hline \multirow{2}{*}{ Worst pattern of invasion } & Others & $2(20 \%)$ & $2(50 \%)$ & \multirow{2}{*}{0.262} \\
\hline & IV/V & $8(80 \%)$ & $2(50 \%)$ & \\
\hline \multirow{2}{*}{ pT stage } & pT2 & $4(15.4 \%)$ & $2(40 \%)$ & \multirow{2}{*}{0.202} \\
\hline & $>\mathrm{pT} 2$ & $22(84.6 \%)$ & $3(60 \%)$ & \\
\hline al otang & pN0 \&pN1 & $11(52.4 \%)$ & $3(60 \%)$ & 0750 \\
\hline pN stage & pN2\&pN3 & $10(47.6 \%)$ & $2(40 \%)$ & 0.159 \\
\hline
\end{tabular}

Figure $1-641$
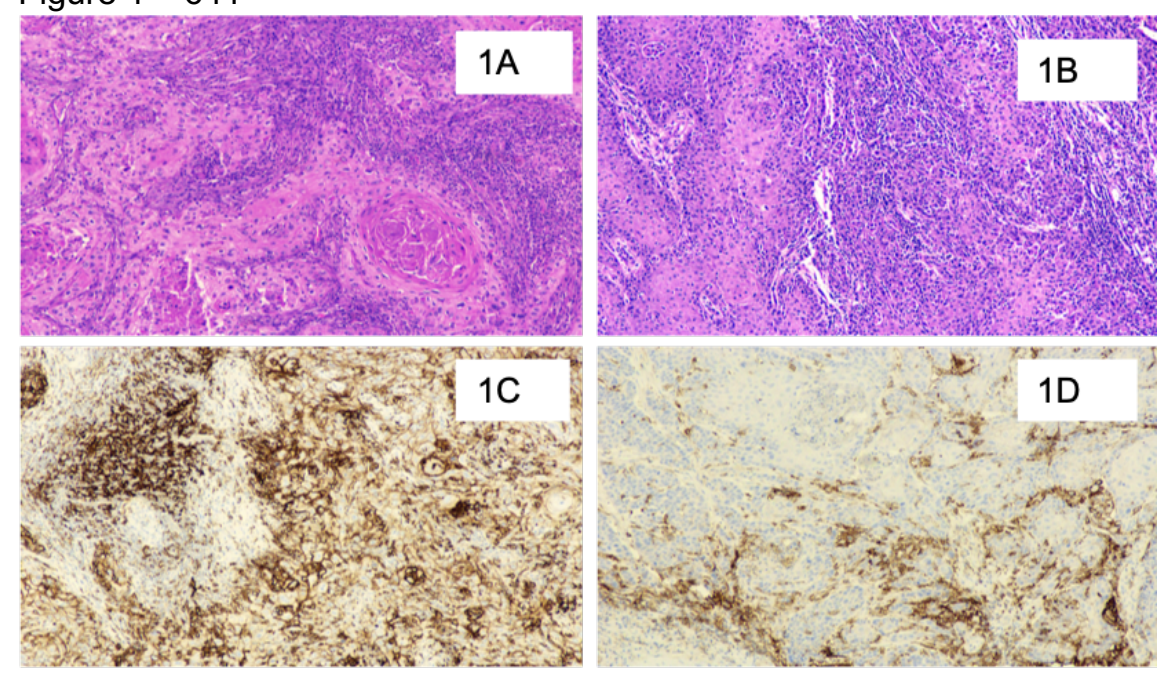

Figure 1A. WDSCC; 1B. MDSCC; 1C. CPS HIGH; ID. CPS LOW 


\section{Conclusions:}

1. Overall PDL-1 percentage positivity in our cohort of HNSCC cases was $84 \%$.

2. We found a statistically significant correlation $(p<0.05)$ between PD-L1 expression and site of PD-L1 evaluation, the histologic grade of the tumor and tumor infiltrating lymphocytes but not with other histologic parameters.

3. Future direction: This is an ongoing study and we are evaluating the outcome and survival of these cases in the PD-L1 high and low expression subgroups.

\section{Whole Slide Analysis of Oral Cavity Cancer: Large Scale Active Learning} Jonathan Folmsbee ${ }^{1}$, Scott Doyle ${ }^{1}$, Margaret Brandwein-Weber ${ }^{2}$

${ }^{1}$ University at Buffalo, SUNY, Buffalo, NY, ${ }^{2}$ Icahn School of Medicine at Mount Sinai, New York, NY

Disclosures: Jonathan Folmsbee: None; Scott Doyle: None; Margaret Brandwein-Weber: None

Background: Deep learning has led to high performance on segmentation tasks in computational pathology. Unfortunately, generating these results requires well annotated, large datasets to train the system to recognize tissue regions. Some training procedures include transfer learning, zero-shot training methods, and semisupervised approaches, however these methods do not utilize feedback loops of human experts. Previously, we have explored the use of Active Learning (AL) as an informed feedback training loop. In this work, we extend our previous work on $A L$ on regions of interest (ROIs) to generate whole slide image (WSI) semantic segmentation of tissue classes. We look to show that AL produces stable classifier improvement on the WSI scale compared to Random Learning (RL).

Design: Our dataset consists of 34 WSIs of oral cavity cancer (OCC) across 33 patients scanned via an Olympus scanner at $40 \mathrm{x}$ magnification $(0.175 \mu \mathrm{m} /$ pixel spatial resolution) and resized to $2 \mu \mathrm{m} /$ pixel. The $\mathrm{AL}$ training procedure (Figure 1) is iterative and performed as follows. ROls with tissue classes of interest were selected from 4 random cases, hand annotated, and used to build our bootstrap training set. A segmentation deep neural network was trained on these annotated samples to generate Version 1 of the model. The remaining unlabeled cases were separated into $2000 \times 2000$ pixel tiles and segmented. Quality assurance (QA) of the segmentation maps was performed by human observers. A new set of 4 ROls from the worst performing images were selected for reannotation and addition to Version 2 of the AL classifier. This was repeated until Version 4. In parallel, at each iteration, a set of random images were selected for annotation to train an RL classifier. The number of images used at each iteration is shown in Table 1. Following each version, the classifier was used to segment 10 holdout WSIs for evaluation.

Results: An example of WSI tissue map results for each version is shown below in Figure 2. We notice that while there is not much of a marked difference in the AL Version 4 and the RL Version 4, we do notice that the AL remained more stable across versions, while $\mathrm{RL}$ was more volatile. 
Figure $1-642$

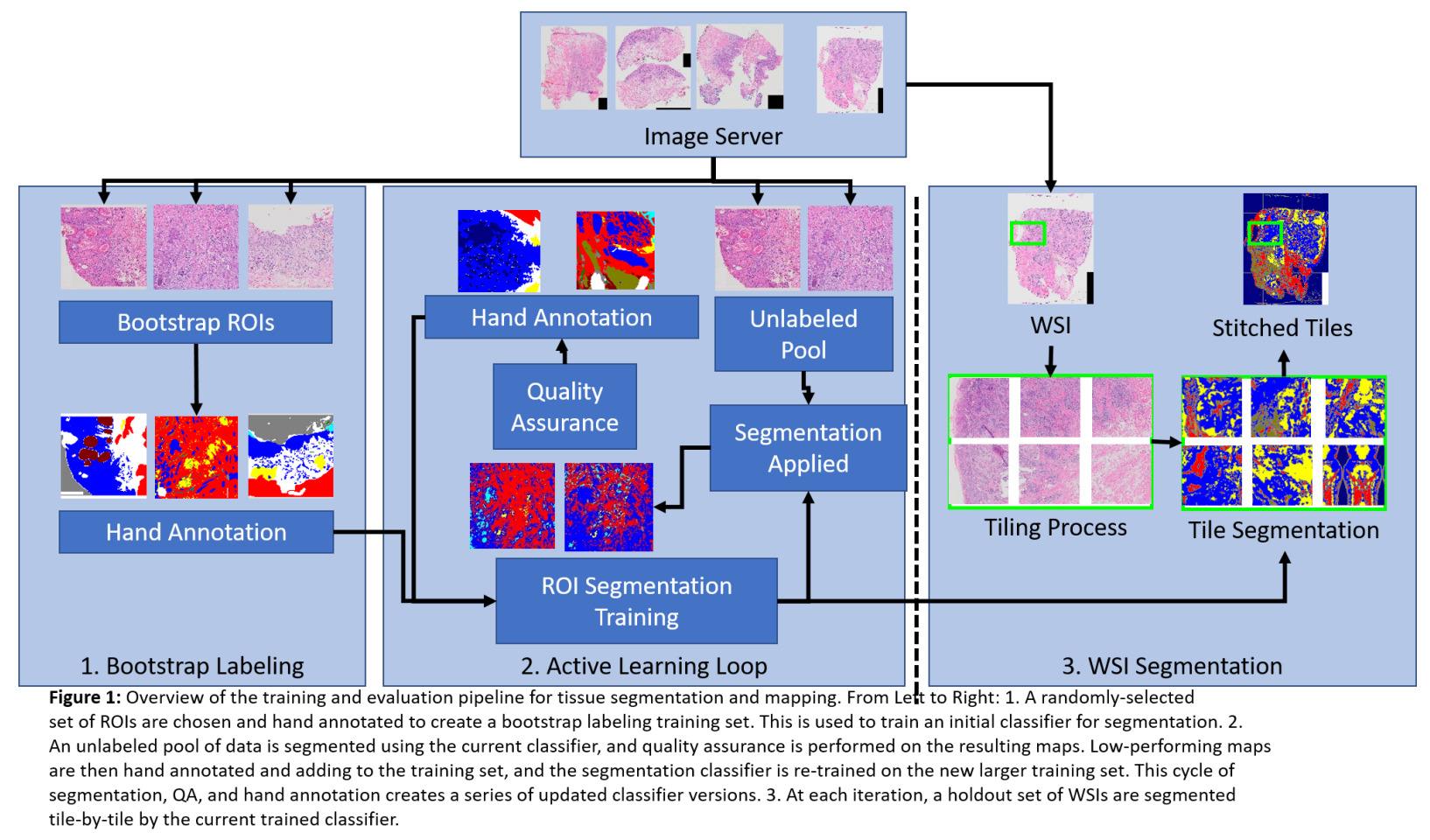

Figure $2-642$

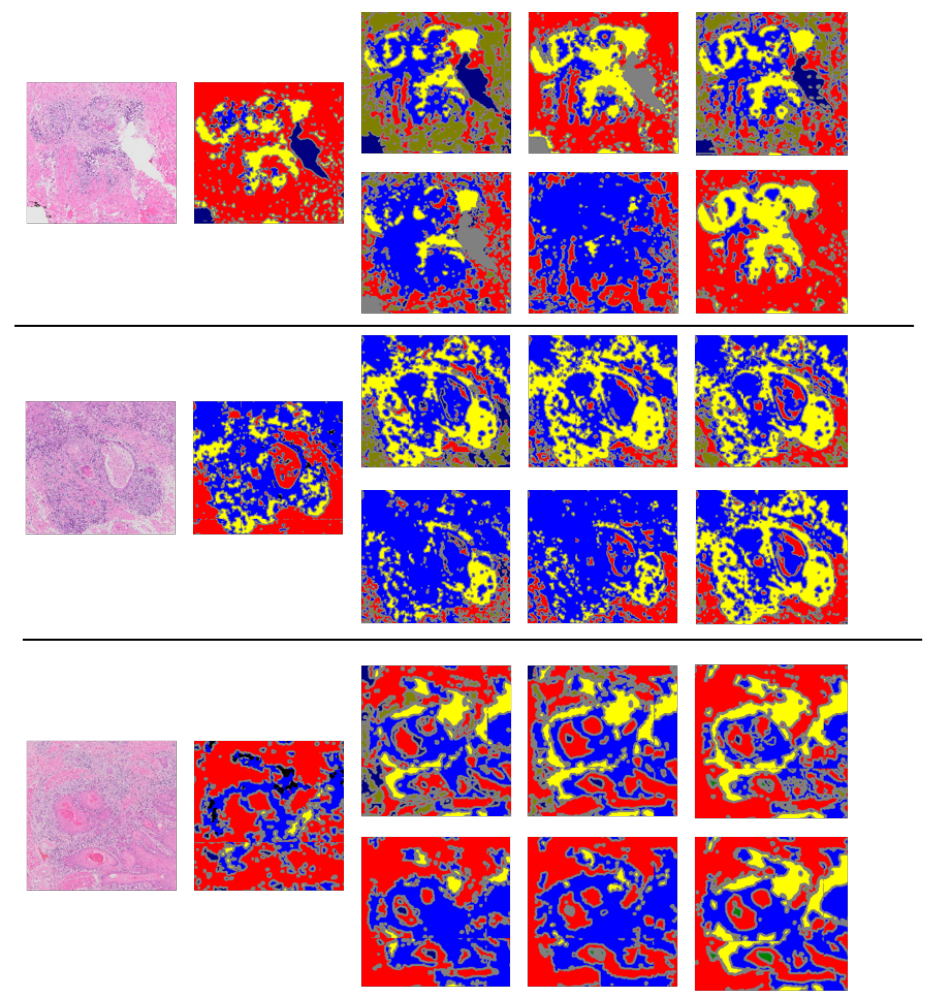

Figure 2: Progression of Segmentation Results. The original H\&E and Version 1 are shown followed by Versions 2-4 of AL in the top rows, and Version 2-4 of RL in the bottom rows. As you can see, AL is more stable across versions than RL. 
Conclusions: The WSI scale segmentation results show more volatility for RL than AL across the dataset. One ground truth annotation used for the AL classifier takes between 90 minutes and 3 hours to generate. Each WSI has on average 25 tiles, meaning the 143 WSI dataset would take between 5362 and 10725 hours to generate ground truth for. An efficient approach to tissue classifier development translates into enormous time savings with respect to QA, meaning the more stable AL approach should mean less time performing QA and annotation.

\section{Glial Fibrillary Acidic Protein Expression Helps Distinguish Pleomorphic Adenoma from Malignant Biphasic Tumours of the Salivary Glands Zuzanna Gorski ${ }^{1}$, Bibianna Purgina ${ }^{1}$, Jason Wasserman ${ }^{2}$ ${ }^{1}$ Ottawa Hospital, University of Ottawa, Ottawa, Canada, ${ }^{2}$ The Ottawa Hospital, Ottawa, Canada}

Disclosures: Zuzanna Gorski: None; Bibianna Purgina: None; Jason Wasserman: None

Background: Pleomorphic adenoma (PA) is the most common type of salivary gland tumour to arise in adults. It is a biphasic tumour composed of both luminal (ductal) cells and abluminal (basal and myoepithelial) cells. Other biphasic salivary gland type tumours, both benign and malignant, can mimic PA, especially on small biopsies. Previous studies have shown that glial fibrillary acidic protein (GFAP) is preferentially expressed in PA and can be useful in the distinction from other salivary gland tumours. However, most of these studies were performed on a small subset of tumour types at time when the classification of salivary gland type tumours was less refined. The purpose of this study was to assess the expression of GFAP in a broad group of both benign and malignant salivary gland tumours.

Design: The expression of GFAP was assessed in 99 salivary gland type tumours include 53 pleomorphic adenomas, 5 basal cell adenomas, 2 myoepitheliomas, 5 adenoid cystic carcinomas, 6 epithelial-myoepithelial carcinomas, 6 mucoepidermoid carcinomas, 7 salivary duct carcinomas, 1 adenocarcinomas NOS, 2 myoepithelial carcinomas, 4 basal cell adenocarcinomas, 5 acinic cell carcinomas and 3 polymorphous adenocarcinomas. Of the malignant cases, 8 were classified as carcinomas ex PA.

Results: GFAP was strongly and diffusely expressed in 50 PAs examined (94\%). Expression was predominantly seen in myoepithelial cells including those in areas of chondromyxoid stroma. GFAP was also strongly and diffusely expressed in 1 basal cell adenoma (20\%) and 1 myoepithelioma (50\%). Rare GFAP positive cells were also seen in 1 basal cell adenocarcinoma (25\%) and 1 polymorphous adenocarcinoma (33\%). Strong expression of GFAP was also seen in 6 cases of carcinoma ex PA (75\%) but only in the residual benign PA component.

Conclusions: Almost all PAs show strong expression of GFAP. In contrast, most malignant neoplasms that can mimic PA on small biopsies show only rare expression. Other benign tumours composed of abluminal cells also show strong expression of GFAP highlighting the spectrum these tumours share with PA. The presence of strong diffuse GFAP expression can favour a benign neoplasm, specifically a PA, on limited tissue biopsy specimens.

\section{PRAME Expression in High-Risk HPV-Positive and Negative Squamous Cell Carcinoma of} the Head and Neck and Regional Lymph Node Metastases

Sarah Gradecki ${ }^{1}$, William Kane ${ }^{1}$, Joseph Coppock ${ }^{1}$, Shyam Raghavan ${ }^{2}$

${ }^{1}$ University of Virginia, Charlottesville, VA, ${ }^{2}$ University of Virginia School of Medicine, Charlottesville, VA

Disclosures: Sarah Gradecki: None; William Kane: None; Joseph Coppock: None; Shyam Raghavan: None

Background: Preferentially expressed antigen in melanoma (PRAME) is a protein in the cancer testis antigen family that can be recognized by cytotoxic T-lymphocytes and acts as a ligand-dependent corepressor of nuclear retinoic acid receptor signaling. PRAME expression has been identified in head and neck squamous cell carcinoma (HNSCC), suggesting that it could be a potential target for immunotherapy. However, no evaluation of PRAME in HPV+ and HPV- HNSCC has been performed. Because HPV+ HNSCC are driven by different mechanisms and have a different prognosis and treatment than HPV- HNSCC, the evaluation of PRAME in each tumor subtype is crucial for understanding the expression patterns and potentially identifying patients who are candidates for PRAME-targeted immunotherapy. 
Design: 78 cases of HNSCC were evaluated in quadruplicate by tissue microarray. PRAME expression was assessed by immunohistochemistry. Staining was scored in tumor cells as $0 \%, 1-25 \%, 26-50 \%, 51-75 \%$, and $>75 \%$. Strength of expression was scored as $0,1+, 2+$, or $3+$. HPV+ vs. HPV- and primary vs. metastatic groups were compared using the Wilcoxon rank-sum test.

Results: Cases included primary HPV- $(\mathrm{N}=35)$, primary HPV+ $(\mathrm{N}=18)$, metastatic HPV- $(\mathrm{N}=14)$, and metastatic $\mathrm{HPV}+(\mathrm{N}=11)$. In total, 43.6\% (34/78) of cases showed nuclear PRAME expression (Figure 1). Overall, significantly more HPV- cases showed PRAME expression as compared to HPV+ cases $(p=0.02)($ Table 1). Among PRAME+ cases, there was a trend toward stronger expression in HPV+ cases as compared to HPV- cases $(p=0.06)$. On subgroup analysis of HPV- cases, metastatic lesions showed significantly more PRAME expression than primary lesions $(p=0.002)$. There was no difference in PRAME expression between HPV+ metastatic and primary SCC $(p=0.36)$.

Table 1: PRAME expression in HPV- and HPV+ primary and metastatic tumors

\begin{tabular}{|c|c|c|c|c|c|c|}
\hline PRAME Expression & $0 \%$ & $1-25 \%$ & $26-50 \%$ & $51-75 \%$ & $>75 \%$ & $P$ value \\
\hline HPV- $(\mathrm{N}=49)$ & $23(46.9)$ & $7(14.3)$ & $4(8.2)$ & $10(20.4)$ & $5(10.2)$ & \multirow{2}{*}{0.02} \\
\hline $\mathrm{HPV}+(\mathrm{N}=29)$ & $21(72.4)$ & $4(13.8)$ & $1(3.5)$ & $1(3.5)$ & $2(6.9)$ & \\
\hline \multicolumn{7}{|l|}{ HPV- } \\
\hline Primary $(\mathrm{N}=35)$ & $21(60.0)$ & $5(14.3)$ & $2(5.7)$ & $5(14.3)$ & $2(5.7)$ & \multirow{2}{*}{0.002} \\
\hline Metastases $(\mathrm{N}=14)$ & $2(14.3)$ & $2(14.3)$ & $2(14.3)$ & $5(35.7)$ & $3(21.4)$ & \\
\hline \multicolumn{7}{|l|}{ HPV+ } \\
\hline Primary $(\mathrm{N}=18)$ & $12(66.7)$ & $3(16.7)$ & $0(0)$ & 1 (5.6) & $2(11.1)$ & \multirow{2}{*}{0.36} \\
\hline Metastases $(\mathrm{N}=11)$ & $9(81.8)$ & $1(9.1)$ & 1 (9.1) & $0(0)$ & $0(0)$ & \\
\hline
\end{tabular}

Figure 1 - 644

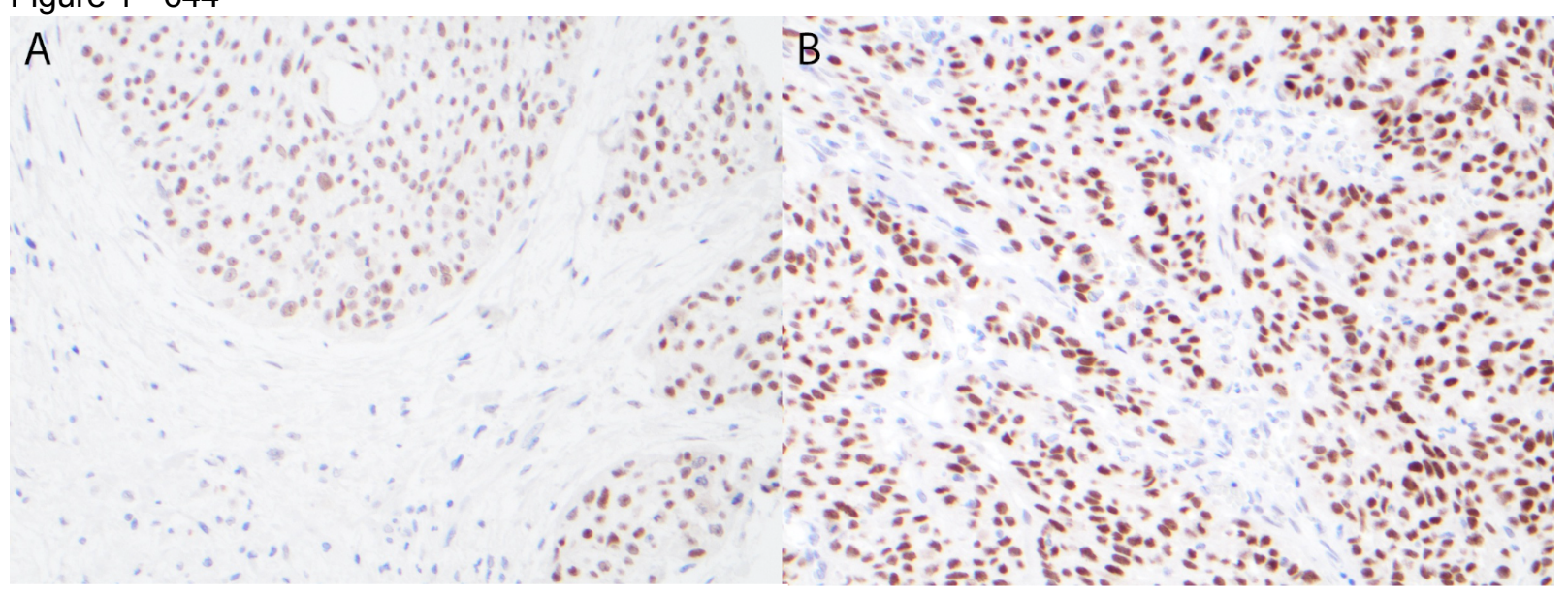

Figure 1: Metastatic HPV- squamous cell carcinoma with diffuse, moderate (2+) nuclear PRAME expression (A, 200x). A rare case of PRAME+ metastatic HPV+ squamous cell carcinoma showing diffuse, strong (3+) nuclear PRAME expression (B, 200x).

Conclusions: PRAME expression was significantly more common in HPV- HNSCC when compared to HPV+ cases. HPV- metastases exhibited greater tumoral PRAME positivity than HPV- primary lesions, suggesting a possible role of progressive PRAME expression in metastases. Additionally, there was a trend toward stronger nuclear PRAME expression in HPV+ tumors. Overall, these findings suggest that the different mechanisms of carcinogenesis in HPV- and HPV+ carcinoma may lead to differential expression of PRAME. Upregulated PRAME in HPV- tumors, particularly HPV- metastatic lesions, may provide a therapeutic target for patients who face a poor prognosis and require aggressive therapy. 
645 Next-Generation Sequencing (NGS) of Salivary Gland Carcinomas (SGC): Correlation with Morphology and Emerging Evidence Supporting a Shift to Molecular Classification

Amin Heidarian ${ }^{1}$, Christine Walko ${ }^{1}$, Bruce Wenig ${ }^{1}$, Juan Hernandez-Prera ${ }^{1}$

${ }^{1}$ H. Lee Moffitt Cancer Center \& Research Institute, Tampa, FL

Disclosures: Amin Heidarian: None; Christine Walko: Consultant, Jackson Genetic Laboratories; Consultant, Intermountain Healthcare; Bruce Wenig: None; Juan Hernandez-Prera: None

Background: Next-generation sequencing (NGS) in salivary gland carcinomas (SGC) is emerging in clinical management based on the identification of targetable mutations to guide systemic therapy. In addition, NGS when correlated with histology, may have diagnostic implications unearthing unusual and heretofore unique associations and previously undescribed findings.

Design: NGS results of 28 SGCs ordered for treatment purposes were analyzed and correlated with morphological findings. Information regarding the biologic and oncogenic effects of gene alterations and levels of evidence for treatment implications were extracted from OncoKB.org and classified as: level 1 (FDA-approved drugs), level 2 (standard care), level 3A (emerging clinical evidence), level 4 (biological evidence), and level R1/R2 (resistance).

Results: Original pathologic diagnoses included: 3 acinic cell carcinomas (ACC), 10 salivary duct carcinomas (SDC), 2 carcinomas ex-pleomorphic adenoma, 1 basal cell adenocarcinoma (BCAC), 1 high-grade mucoepidermoid carcinoma (MEC), and 11 SGCs not otherwise specified (NOS). Twenty-seven cases (97\%) had at least one gene alteration that added to a total of 83 alterations with biologic and oncogenic effects. The level of evidence for treatment was: 10 level 1, 2 level 2, 8 level 3A, 7 level 4, and 2 level R1/R2. Following correlation with morphology, in 6 cases (22\%) the NGS had diagnostic implications: 1 ACC and 1 intrathyroidal SGC NOS were reclassified as secretory carcinoma due to an ETV6-NTRK3 fusion; RET-NCOA4 fusions were detected in two widely metastatic cases originally diagnosed as SDC and SGC NOS, resulting in reclassification as intercalated duct adenocarcinoma; NOTCH1 and KDM6A co-mutations were found in 1 SGC NOS suggesting the diagnosis of adenoid cystic carcinoma (MYB confirmation pending); and 1 SGC NOS with sebaceous differentiation was reclassified as a high-grade variant of epithelial-myoepithelial carcinoma due to an HRAS mutation. Unusual findings that require further investigation include BCOR mutation in a BCAC and SDCs with $M D M 2 / C D K 4$ amplification, $C D H 1$ mutation with loss of E-cadherin by immunohistochemistry (IHC), or BRAFV600E mutation confirmed by IHC. The latter case had an unusual squamoid morphology and nuclear features reminiscent of papillary thyroid carcinoma.

Conclusions: NGS of SGCs may provide clinicians with information about gene alterations potentially guiding treatment options. Moreover, in $22 \%$ of cases, the NGS findings can be leveraged to improve tumor classification. To this end, we provide evidence of the existence of a widely metastatic RET-NCOA4 positive intercalated duct adenocarcinoma. Advances in NGS and its increasing use in cancer care result in novel findings that challenge long-standing concepts in salivary gland cancer diagnosis and provide emerging evidence to consider the need for molecular classification of SGC.

\section{Sclerosing Polycystic Adenoma: Conclusive Clinical and Molecular Evidence of Its Neoplastic Nature \\ Juan Hernandez-Prera ${ }^{1}$, Amin Heidarian ${ }^{1}$, Bruce Wenig ${ }^{1}$ \\ ${ }^{1}$ H. Lee Moffitt Cancer Center \& Research Institute, Tampa, FL}

Disclosures: Juan Hernandez-Prera: None; Amin Heidarian: None; Bruce Wenig: None

Background: Sclerosing polycystic adenosis, initially considered a non-neoplastic salivary gland lesion and classified as such in the 2017 WHO classification of head and neck tumors, has been the subject of controversy regarding its possible neoplastic nature. The reporting of recurrent PTEN mutations and PI3K pathway alteration in these lesions represent evidence to support these lesions as being neoplastic and more appropriately referred to as sclerosing polycystic adenoma (SPA). Herein, we provide additional evidence that support the classification of SPAs as a true neoplasm. 
Design: Eight cases of SPA were identified in our database and consultation files. Six cases were subjected to PTEN immunohistochemistry (IHC) and 2 subjected to next-generation sequencing (NGS). In addition, one patient underwent genetic counseling and germline testing.

Results: The cases included 5 men and 3 women with a mean age of 41 years (range 12-78) and all tumors arose in the parotid gland. One patient had multiple recurrences over a period of 2 years. Morphologically the tumors were circumscribed and characterized by an admixture of acini, ducts and cysts embedded in fibrotic/sclerotic stroma. One case had stromal lipomatous changes. The cells lining the ducts and cysts showed variable granular, vacuolated, foamy and apocrine cytoplasmic features, while in the majority of cases the acinar cells contained brightly eosinophilic granules. The intraductal proliferations showed focal mild to moderate atypia in 3 cases. One case showed overt malignant morphology that ranged from intraductal carcinoma to invasive carcinoma with apocrine ductal phenotype. Cases tested for PTEN IHC showed loss of nuclear expression in the acinar and ductal cells with retained PTEN expression in the myoepithelial cell and stroma. NGS

detected PTEN and PIK3R1 mutations in both cases, however, one case had an additional different PTEN mutation. The patient with two PTEN alterations had clinical stigmata of Cowden syndrome which was confirmed with germline genetic testing.

Conclusions: Our findings herein documented including recurrence of tumor, malignant transformation, high prevalence of PTEN alterations and possible hitherto undescribed association with Cowden syndrome add support to classifying these lesions as true neoplasms and justify the designation of SPA.

\section{Immunohistochemical Correlates of the Recently Described Molecular Landscape of Olfactory Neuroblastoma Highlight Prognostic and Therapeutic Targets \\ Alexandra Isaacson ${ }^{1}$, Anand Rajan KD ${ }^{1}$, Jason Hornick ${ }^{2}$, Andrew Bellizzi ${ }^{1}$ \\ ${ }^{1}$ University of lowa Hospitals \& Clinics, lowa City, IA, ${ }^{2}$ Brigham and Women's Hospital, Harvard Medical School, Boston, MA}

Disclosures: Alexandra Isaacson: None; Anand Rajan KD: None; Jason Hornick: None; Andrew Bellizzi: None

Background: Olfactory neuroblastoma (ONB) is an aggressive neuroendocrine neoplasm (NEN) of the nasal cavity with a propensity for late recurrence and no targeted therapeutic options. The first "multiomics" ONB study identified distinct neural $(60 \%)$ and basal $(40 \%)$ molecular subtypes, which correlated with grade and outcome. Potentially actionable IDH2 mutations have been reported in 5-17\% and FGFR3 amplification was recently found in $28 \%$. Our neuroendocrine program has a special interest in SSTR2A and CXCR4, which are peptide receptor radionucleotide therapy targets. We sought to immunohistochemically (IHC) confirm the recently described molecular genetic landscape of ONB, with prognostic and therapeutic implications.

Design: Synaptophysin (Syn), chromogranin A (CgA), AE1/AE3, S-100, SOX10, KIT, Ki-67, beta-catenin, cyclin D1, SSTR2A, FGFR3, CXCR4, and IDH1/2 multi-mutation specific IHC were performed on tissue microarrays (triplicate $1 \mathrm{~mm}$ cores) of 39 ONBs from 29 patients. IHC was evaluated for intensity (0-3+) and extent (0-100\%) with an $\mathrm{H}$-score calculated. S-100 staining in sustentacular cells was absent (0) or present, quantified as $1+$ (up to 4 per 20x field), 2+ (5-19), 3+ (20+). Ki-67 index was assessed visually and by digital analysis, with a manual count performed in discrepant cases. Beta-catenin was assessed for the presence of nuclear staining. FGFR3 staining was reported as absent $(0)$ or present $(1-3+)$. IDH1/2 staining was evaluated from $0-3+(0-1=$ negative, 2=equivocal, 3=positive). Glass slides were reviewed with a Hyams grade assigned, and charts were reviewed for Kadish-Morita stage, duration of follow-up, and vital status. Fisher's exact and Kruskal-Wallis tests and KaplanMeier curves were generated with $p<0.05$ considered significant.

Results: Neural phenotype (Syn, $\mathrm{CgA}+$ ) correlated with lower grade, while basal phenotype (AE1/AE3, KIT+) correlated with higher grade (Table). Ki-67 index increased with Hyams grade. SSTR2A and CXCR4 were preferentially expressed in low and high-grade tumors, respectively. No tumors demonstrated nuclear beta-catenin. Six tumors (17\%) demonstrated strong cyclin D1 expression (H-score>200; suggesting amplification); 8 (28\%) demonstrated moderate to strong (2-3+) staining for FGFR3. One tumor (4\%) was IDH1/2positive. Median follow-up was $46 \mathrm{mo} ; 16$ patients had died at last follow-up. Any KIT-positivity predicted worse survival (16 mo vs $158 \mathrm{mo}, \mathrm{p}=0.02)$ (Figure), while neural phenotype (Syn/CgA H-score>100) was associated with more favorable prognosis (116 mo vs. $28 \mathrm{mo} ; \mathrm{p}=0.03$ ). 


\begin{tabular}{|c|c|c|c|c|c|}
\hline \multicolumn{2}{|l|}{ Marker } & $\begin{array}{l}\text { Hyams Grade } 1 \& 2 \\
(n=17)\end{array}$ & $\begin{array}{l}\text { Hyams Grade } 3 \\
(n=13)\end{array}$ & $\begin{array}{l}\text { Hyams Grade } 4 \\
(n=9)\end{array}$ & $P$ value \\
\hline Syn & $\begin{array}{l}\text { \% positive } \\
\text { Mean } \\
\text { (median) }\end{array}$ & $\begin{array}{l}100 \% \\
254(300)\end{array}$ & $\begin{array}{l}77 \% \\
244(300)\end{array}$ & $\begin{array}{l}56 \% \\
31(25)\end{array}$ & $\begin{array}{l}p=0.0124 \\
p=0.0003\end{array}$ \\
\hline $\mathrm{CgA}$ & $\begin{array}{l}\text { \% positive } \\
\text { Mean } \\
\text { (median) }\end{array}$ & $\begin{array}{l}100 \% \\
224(260)\end{array}$ & $\begin{array}{l}77 \% \\
238(300)\end{array}$ & $\begin{array}{l}33 \% \\
25(17)\end{array}$ & $\begin{array}{l}p=0.0009 \\
p=0.0004\end{array}$ \\
\hline AE1/AE3 & $\begin{array}{l}\text { \% positive } \\
\text { Mean } \\
\text { (median) }\end{array}$ & $\begin{array}{l}31 \% \\
9(3)\end{array}$ & $\begin{array}{l}45 \% \\
93(98)\end{array}$ & $\begin{array}{l}100 \% \\
107(103)\end{array}$ & $\begin{array}{l}p=0.0425 \\
p=0.0002\end{array}$ \\
\hline S-100 & $\begin{array}{l}\text { \% positive } \\
\text { Mean } \\
\text { (median) }\end{array}$ & $23 \%$ & $\begin{array}{l}40 \% \\
2(2)\end{array}$ & $\begin{array}{l}22 \% \\
2(2)\end{array}$ & $\begin{array}{l}p=0.0946 \\
p=0.090\end{array}$ \\
\hline sox10 & $\begin{array}{l}\text { \% positive } \\
\text { Mean } \\
\text { (median) }\end{array}$ & $\begin{array}{l}38 \% \\
7(5)\end{array}$ & $\begin{array}{l}22 \% \\
8(8)\end{array}$ & $\begin{array}{l}50 \% \\
81(18)\end{array}$ & $\begin{array}{l}p=1.000 \\
p=0.4626\end{array}$ \\
\hline KIT & $\begin{array}{l}\text { \% positive } \\
\text { Mean } \\
\text { (median) }\end{array}$ & $\begin{array}{l}6 \% \\
195(195)\end{array}$ & $\begin{array}{l}50 \% \\
175(167)\end{array}$ & $\begin{array}{l}88 \% \\
195(185)\end{array}$ & $\begin{array}{l}p=0.0004 \\
p=0.0008\end{array}$ \\
\hline $\begin{array}{l}\text { Ki-67 PI } \\
(\%)\end{array}$ & $\begin{array}{l}\text { Mean } \\
\text { (median) }\end{array}$ & $9.1(3.8)$ & $19(6)$ & $46.5(50.8)$ & $p=0.0055$ \\
\hline SSTR2A & $\begin{array}{l}\text { \% positive } \\
\text { Mean } \\
\text { (median) }\end{array}$ & $\begin{array}{l}94 \% \\
209(240)\end{array}$ & $250(300)$ & $\begin{array}{l}22 \% \\
58(58)\end{array}$ & $\begin{array}{l}p=0.0227 \\
p=0.0004\end{array}$ \\
\hline CXCR4 & $\begin{array}{l}\text { \% positive } \\
\text { Mean } \\
\text { (median) }\end{array}$ & $\begin{array}{l}33 \% \\
163(173)\end{array}$ & $\begin{array}{l}67 \% \\
106(59)\end{array}$ & $\begin{array}{l}100 \% \\
207(207)\end{array}$ & $\begin{array}{l}p=0.0105 \\
p=0.0012\end{array}$ \\
\hline
\end{tabular}

Figure $1-647$

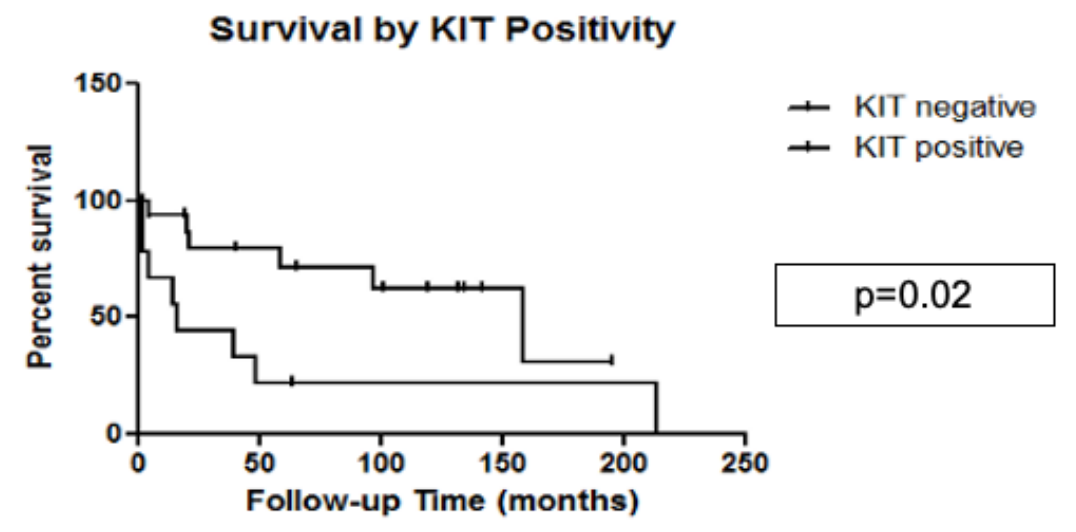

Conclusions: Our findings validate the recently described molecular landscape of ONB, including prognostically favorable neural and adverse basal subtypes and potentially actionable FGFR3 overexpression and (rare) IDH mutation. We also identify frequent expression of the biotheranostic targets SSTR2A and CXCR4. 


\section{Evaluation of Head and Neck Paragangliomas Using Immunohistochemical Surrogates for SDHx and ATRX Molecular Testing}

Aanchal Kakkar ${ }^{1}$, Diya Roy ${ }^{1}$, Kavneet Kaur ${ }^{1}$, Nikita Birua ${ }^{1}$, Alok Thakar ${ }^{1}$

${ }^{1}$ All India Institute of Medical Sciences, New Delhi, India

Disclosures: Aanchal Kakkar: None; Diya Roy: None; Kavneet Kaur: None; Nikita Birua: None; Alok Thakar: None

Background: Paragangliomas (PGL) have highest degree of heritability among all human cancers. Head and neck (HN) PGL account for majority of extra-adrenal PGL. SDHx mutations are the most frequent genetic event in HNPGL, in familial as well as apparently sporadic cases. SDHB immunohistochemistry is a reliable and economical tool for detecting $S D H x$ mutant cases. Recently, whole exome sequencing identified somatic ATRX mutations in a subset of SDHx mutant PGLs. We therefore aimed to evaluate a large cohort of HNPGLs

for SDHx and ATRX mutations using established immunohistochemical surrogates.

Design: HNPGLs diagnosed between 2014 and 2019 were retrieved, histomorphological features reviewed, and SDHB and ATRX immunohistochemistry performed. SDHB staining was categorized as retained, lost, or weak cytoplasmic, with latter two considered as SDH-deficient; ATRX expression was reported as lost or retained.

Results: Sixty-five HNPGL were identified (mean age:37.6years; range:9-69 years; M:F=0.55) and distributed as follows: 35 (53.9\%) carotid body, 22 (33.8\%) jugulotympanic, 7 (10.8\%) vagal, and one (1.5\%) sinonasal PGL.

SDHB staining was interpretable in 62 cases. Twenty-two cases (35\%) were SDH-deficient: complete loss in 11 cases $(17.7 \%)$, and weak cytoplasmic in 11 cases (17.7\%). SDH-deficient HNPGL were commoner in males $(48 \%$ vs $28 \%$ ), and accounted for $6 / 7(85.7 \%)$ bilateral/multifocal cases, $50 \%$ of cases with metastases, and two patients with positive family history (100\%). There was no significant difference in histomorphology between SDH-retained and SDH-deficient HNPGL. All 22 SDH-deficient HNPGL showed retained ATRX expression.

Conclusions: Significant proportion of HNPGL are SDH-deficient on SDHB immunohistochemistry. These SDHdeficient HNPGL are associated with multiple tumors, metastases, and positive family history. ATRX loss was not identified in any of the SDH-deficient PGLs, possibly indicating low prevalence of ATRX mutations. These findings need further validation by sequencing (work in progress) for an in-depth appraisal of the genetic features of HNPGL.

\section{Novel Possible Driver Genes in Vagal Paragangliomas}

Dmitry Kalinin ${ }^{1}$, Vladislav Pavlov², Elena Lukyanova², Maria Fedorova², Elena Pudova², Alexander Golovyuk $^{3}$, George Krasnov², Anastasiya Snezhkina², Anna Kudryavtseva ${ }^{2}$

${ }^{1}$ A. V. Vishnevsky National Medical Research Center of Surgery of the Russian Ministry of Healthcare, Moscow, Russia, ${ }^{2}$ Engelhardt Institute of Molecular Biology, Russian Academy of Sciences, Moscow, Russia, ${ }^{3}$ Vishnevsky Institute of Surgery, Ministry of Health of the Russian Federation, Moscow, India

Disclosures: Dmitry Kalinin: None; Vladislav Pavlov: None; Elena Lukyanova: None; Maria Fedorova: None; Elena Pudova: None; Alexander Golovyuk: None; George Krasnov: None; Anastasiya Snezhkina: None; Anna Kudryavtseva: None

Background: Vagal paragangliomas (VPGLs) are rare neuroendocrine neoplasms of the head and neck that arise from paraganglionic tissue located along the vagus nerve. VPGLs occur both as familial and sporadic forms.

Hereditary VPGLs can be associated with germline mutations in known susceptibility genes, such as $S D H x, R E T$, VHL, NF1, MEN2, MAX, TMEM127, FH, and SLC25A11, when somatically mutated genes mainly remain elusive. Identification of genes involved in somatic activation of tumor development is important for the understanding of the molecular mechanisms underlying the VPGL pathogenesis, as well as improving tumor screening and management.

Design: Whole exome sequencing of 15 paired tumor and normal (blood or lymph node) tissues obtained from Russian patients with VPGLs was performed on an Illumina platform. Based on these data, somatic mutation 
spectra were found for each sample. The MutSigCV software was used to identify significantly somatic mutated genes in VPGLs.

Results: VPGLs were found to be characterized by low somatic mutation frequency. A total of 22 significantly mutated somatic genes $(\mathrm{p} \leq 0.05)$ were identified: BCAS4, RBM3, SDHD, EFCAB9, SPDYE4, MRPL46, ATG5, SUMO1, APOL2, CSRP2, BTG3, ORMDL3, CHTF8, AGTR2, FTHL17, PMEPA1, RNF146, RNF138, MRPL42, LIAS, WNT7B, and TIMM22. The most significant results were obtained for two genes, BCAS4 $(\mathrm{p}=0.0003)$ and RBM3 ( $\mathrm{p}=0.0005$ ), which have not been previously shown to be involved in the pathogenesis of paragangliomas or pheochromocytomas. However, both genes were reported as cancer-associated. SDHD gene, which is a widely known driver gene in paragangliomas and pheochromocytomas that often carries germline mutations, was also identified in this study. These results confirm that SDHD might also be altered at the somatic level and play an important role in tumorigenesis of VPGLs.

Conclusions: Our study first revealed genes with a high rate of somatic mutations in VPGLs. These genes can be considered as possible driver genes, in which somatic mutations confer a selective advantage to cells and contribute to tumor development.

This study was financially supported by the Russian Science Foundation, grant no. 19-15-00419.

\section{Combination of Computerized Patterns of Tumor Multinucleation and Tumor-Infiltrating Lymphocytes on H\&E Images is Prognostic for Overall and Disease-Free Survival in p16 positive Oropharyngeal Squamous Cell Carcinoma Patients Including AJCC 8th Edition Stage Groups: A Multi-Site Study \\ Can Koyuncu', German Corredor ${ }^{2}$, Cheng Lu², Paula Toro², Pingfu Fu², Shlomo Koyfman², Deborah Chute $^{3}$, David Adelstein ${ }^{3}$, Wade Thorstad ${ }^{4}$, Justin Bishop ${ }^{5}$, Farhoud Faraji ${ }^{6}$, James Lewis ${ }^{7}$, Anant Madabhushi ${ }^{2}$ \\ ${ }^{1} \mathrm{CCIPD}$, Case Western Reserve University, Cleveland, $\mathrm{OH},{ }^{2}$ Case Western Reserve University, Cleveland, $\mathrm{OH},{ }^{3}$ Cleveland Clinic, Cleveland, $\mathrm{OH},{ }^{4}$ Washington University in St. Louis, St. Louis, MO, ${ }^{5}$ UTSouthwestern Medical Center, Dallas, TX, ${ }^{6}$ University of California, San Diego, San Diego, CA, ${ }^{7}$ Vanderbilt University Medical Center, Nashville, TN}

Disclosures: Can Koyuncu: None; German Corredor: None; Cheng Lu: None; Paula Toro: None; Pingfu Fu: None; Deborah Chute: None; David Adelstein: None; Wade Thorstad: Employee, Elekta Inc; Justin Bishop: None; Farhoud Faraji: None; James Lewis: None; Anant Madabhushi: Advisory Board Member, Aiforia Inc; Primary Investigator, Bristol Myers-Squibb; Primary Investigator, Astrazeneca

Background: Oropharyngeal squamous cell carcinoma (OPSCC) patients can have major morbidity from current treatment regimens and a subset of patients suffer disease progression despite treatment, necessitating accurate identification of patients with aggressive versus indolent tumors. In this study, we sought to evaluate whether the combination of computer extracted features of tumor cell multinucleation (MN) and spatial interplay of tumorinfiltrating lymphocytes (TILs) is prognostic of overall (OS) and disease free (DFS) survival in p16 positive OPSCC patients including in individual AJCC 8th edition staging groups.

Design: OPSCC H\&E slides from 967 patients were retrospectively collected from 6 different sites. 168 patients from site 1 formed the training set (D1) and the remaining five sites formed the validation set (D2, $n=799$ $(322+94+120+158+105))$.

A deep learning model was employed to automatically calculate a MN risk index (MuNI), which is the density of MN within epithelial regions, for each patient. A separate ML model was also used to measure a risk score related to the interplay between TILs and tumor cells (SpaTIL). A random survival forest estimator was then trained using $\mathrm{MuNI}$ and SpaTIL associated risk scores to compute the final risk score. The median value of the risk scores in D1 were used to dichotomize patients in D2 as either low- or high-risk.

Results: In D2, as a baseline, MuNI alone and SpaTIL alone yielded HR=1.77 (95\% Cl:1.33-2.35, p<0.0001, Cindex:0.60) and $\mathrm{HR}=1.53(95 \% \mathrm{Cl}: 1.15-2.03,<0.03,0.60)$ for predicting $\mathrm{OS}$ and $1.67(1.3-2.14, \mathrm{p}<0.0001)$ and 1.36 $(95 \% \mathrm{Cl}: 1.06-1.75,<0.01)$ for predicting DFS, respectively. For the combined MuNI and SpaTIL classifier, patients 
with high-risk scores had worse survival than for the classifiers individually, yielding HR=2.14 (95\% Cl:1.58-2.89, $p<0.0001$, C-index:0.64) for predicting OS and $1.93(1.48-2.52,<0.0001)$ for predicting DFS. HRs and $p$-values for predicting OS and DFS within individiual AJCC 8th editing stage groups were presented in Table 1. C-Index scores were found to be statistically significantly different for the individual biomarkers compared to their combination with p-values 0.02 for SpaTIL vs the combination and 0.05 for MuNI vs the combination [1].

\begin{tabular}{|c|c|}
\hline & Stage-I (n:392) \\
\hline SpaTIL alone & $1.41(0.87-2.28,0.15,0.59)$ \\
\hline MuNI alone & $1.77(1.10-2.86,0.02,0.59)$ \\
\hline MuNI+SpaTIL & $2.02(1.18-3.45,0.003,0.66)$ \\
\hline SpaTIL alone & $1.21(0.80-1.83,0.37)$ \\
\hline MuNI alone & $1.9(1.25-2.88,0.002)$ \\
\hline MuNI+SpaTIL & $1.98(1.24-3.16,0.001)$ \\
\hline
\end{tabular}

HR (95\% Cl, p-value, C-Index) for predicting OS

\begin{tabular}{|c|c|}
\hline Stage-II (n:224) & Stage-III (n:161) \\
\hline $2.02(1.23-3.33,0.01,0.61)$ & $1.04(0.624-1.75,0.87,0.54)$ \\
\hline $1.41(0.85-2.32,0.19,0.61)$ & $2.05(1.22-3.43,0.01,0.59)$ \\
\hline $1.9(1.12-3.23,0.009,0.64)$ & $1.91(1.13-3.21,0.01,0.58)$ \\
\hline \multicolumn{2}{|c|}{$\mathrm{IR}(95 \% \mathrm{Cl}, \mathrm{p}$-value) for predicting DFS } \\
\hline $1.72(1.11-2.65,0.02)$ & $1.00(0.63-1.59,0.99)$ \\
\hline $1.12(0.72-1.72,0.62)$ & $2.02(1.27-3.22,0.005)$ \\
\hline $1.62(1.02-2.57,0.03)$ & $1.66(1.04-2.64,0.03)$ \\
\hline
\end{tabular}

Conclusions: We were able to show improved prognostication in $\mathrm{p} 16$ positive OPSCC patients by combining features relating to multinucleation, and the spatial arrangement of TILs. The combined method was found to be statistically significant for predicting OS and DFS for each AJCC 8th edition staging group and to be statistically significantly better than MuNI and SpaTIL alone.

[1] Kang L, Chen W, Petrick NA, and Gallas BD. (2014) Comparing two correlated C indices with right-censored survival outcome: a one-shot nonparametric approach. Statistics in Medicine, 34(4), 685-703, doi:

$10.1002 / \operatorname{sim} .6370$

\section{Human Papillomavirus-Positive Preneoplasia of the Oropharynx}

Silva Kristo ${ }^{1}$, Uday Shergill ${ }^{2}$, Mihai Merzianu ${ }^{3}$

${ }^{1}$ University at Buffalo, SUNY, Buffalo, NY, ${ }^{2}$ Jackson Memorial Hospital/University of Miami Hospital, Miami, FL, ${ }^{3}$ Roswell Park Comprehensive Cancer Center, Buffalo, NY

Disclosures: Silva Kristo: None; Uday Shergill: None; Mihai Merzianu: None

Background: Human papillomavirus-associated oropharyngeal squamous cell carcinoma (HPV OPSCC) involves tonsillar crypts and often presents with neck metastases. Most HPV oropharyngeal neoplasis is regarded by convention as invasive. HPV-positive carcinoma in situ (HPV OPCIS) was reported in nonneoplastic tonsils and HPV-positive oral epithelial dysplasia (HPV OED) was well documented in oral cavity but HPV OPCIS is less understood due to crypt epithelium histoanatomy and discontinuous basement membrane. We describe here a cohort of HPV OPCIS associated with HPV OPSCC.

Design: Twenty-four OPCIS samples (14 resections, 10 biopsies) and a control group of OPSCC radical tonsillectomies without reported CIS $(n=9)$ were assessed for dysplasia, subsite distribution and HPV status by p16 and/or HPV DNA 16/18 in situ hybridization. Only cases of high grade dysplasia/CIS were included, defined by abrupt transition from adjacent nonneoplastic mucosa, non-contiguity with the invasive component, absence of "surface colonization" and sharp demarcation or an identifiable basement membrane. CIS histologic type, its extent and localization were documented.

Results: OPSCC from 27 men and 6 women, mean age 58 (range, 41-80), involving palatine $(n=27)$ and lingual $(n=6)$ tonsils, soft palate $(n=5)$, lateral wall and vallecula ( 1 each) were included. Tumor involved multiple OP subsites in 7 cases, two of which involved hard palate and posterolateral tongue.

HPV was positive in 19 of 24 selected cases and in all 9 control OPSCC. Of 5 HPV-negative OPSCC, 4 were keratinizing and one nonkeratinizing. OPCIS was confirmed in all 24 selected cases (10 focal, 5 multifocal, 9 
extensive) and identified in 4 controls ( 3 focal, 1 multifocal), overall involving the surface $(n=13)$, crypt $(n=7)$ or both $(n=8)$ epithelia. 21 OPCIS were HPV-positive (16 nonkeratinizing, 3 mixed and 1 keratinizing ) and 3 were HPVnegative (all 3 keratinizing).

The invasive and in situ components were adjacent in all cases, sharing the histology in 27 of 28 cases and HPV status in 26 of 28 cases. Two cases had HPV OPCIS but HPV-negative OPSCC: one incidental focus of tonsillar basaloid CIS was identified adjacent to an HPV-negative keratinizing OSCC of posterolateral tongue and one tongue base SCC showed similar nonkeratinizing morphology but the invasive component was p16 negative.

(Figure 1)

Overall, 26 of 32 tumors had neck metastases but none of the 3 cases with extensive OPCIS and microinvasion only (including 2 HPV positive) did. One soft palate HPV OPCIS recurred locally 4 years later without frank invasion. (Figure 1)
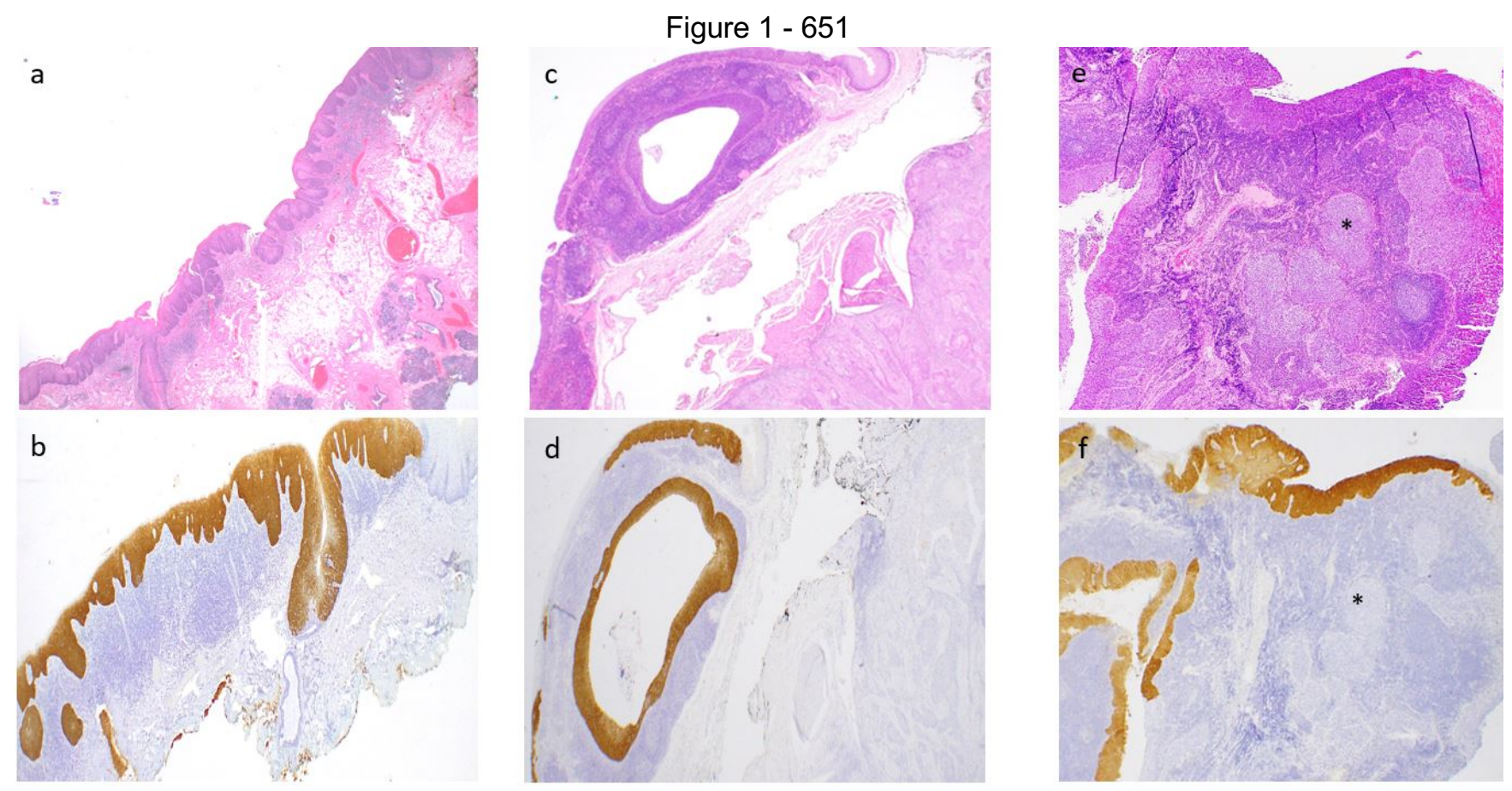

Figure 1. Unusual oropharyngeal HPV-associated carcinoma in situ, all with basaloid morphology: (a) Extensive HPV-positive CIS with microinvasion only involving soft and hard palate mucosa. HPV CIS recurred 4 years later without frank invasion. (b) Minor salivary gland duct involvement and sharp circumscription from the non-neoplastic mucosa (right upper corner) are best highlighted by p16. (c,d) Incidental p16 HPV CIS involving the lingual tonsil surface and crypt epithelium mucosa detected in a glossectomy for conventional p16 negative SCC (right lower). (e, f) Typical HPV-associated tonsillar nonkeratinizing SCC $\left({ }^{*}\right)$ is p16-negative. Only the adjacent CIS involving both surface and crypt epithelium is p16 positive. 

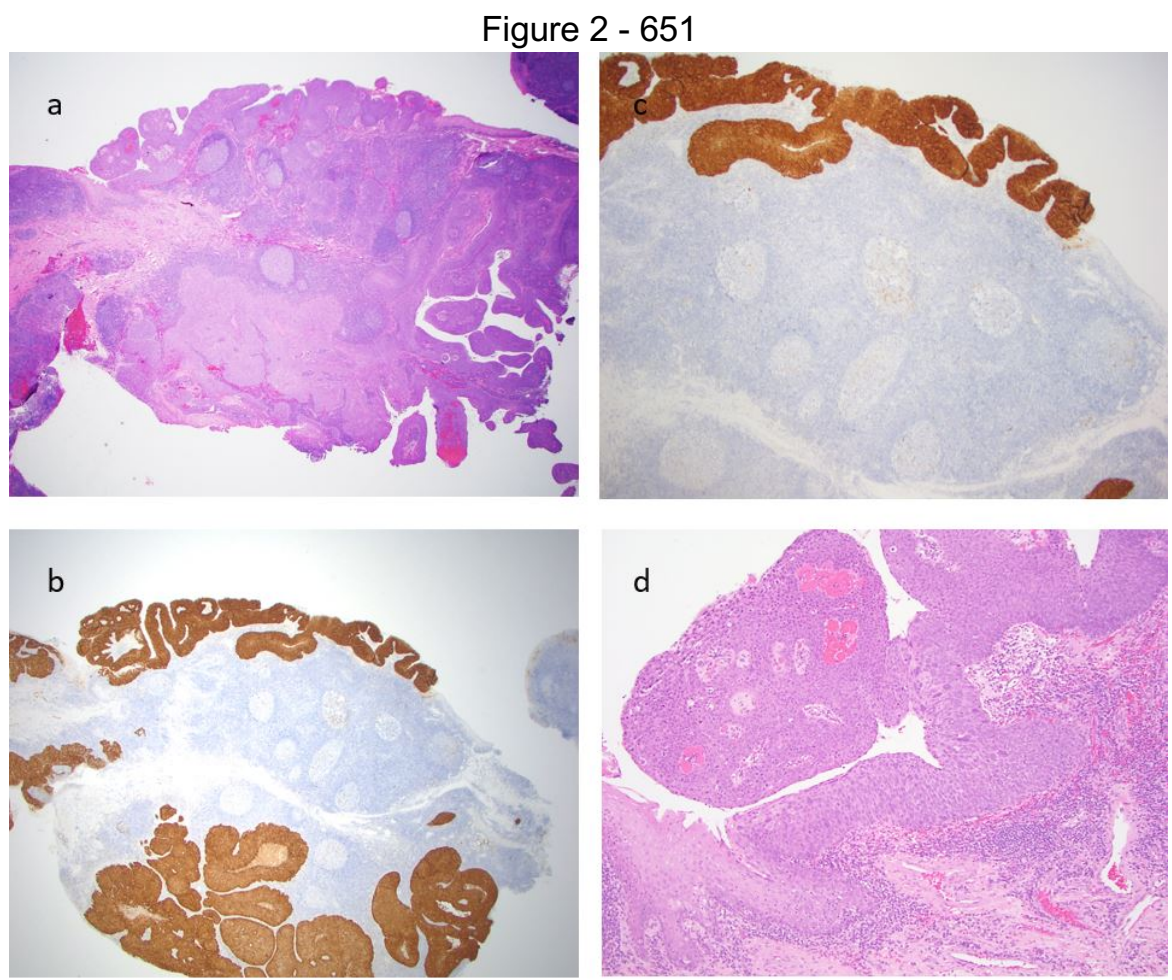

Figure 2. Typical tonsillar HPV OPSCC with extensive CIS surface involvement, sharply delineated from adjacent mucosa (hematoxylin and eosin X20 [a]) and with superficial crypt involvement best highlighted by p16 immunohistochemistry (X40 [b]; X100 [c]). Typical nonkeratinizing CIS with viral-induced atypia (hematoxylin and eosin, X200 [d]).

Conclusions: HPV OPCIS is rare but likely under recognized, usually focal, involving surface and crypt epithelium, and has similar phenotype with the adjacent HPV OPSCC in most cases.

A subset of HPV OPCIS with extensive surface involvement and minimal or no invasion should be recognized since it resembles HPV OED and could be managed conservatively.

\section{The Utility of Next Generation Sequencing in Histologically Challenging Head and Neck Carcinomas \\ Hannah Lee ${ }^{1}$, Roberto Ruiz-Cordero ${ }^{1}$, Annemieke van Zante ${ }^{1}$ \\ ${ }^{1}$ University of California, San Francisco, San Francisco, CA}

Disclosures:vHannah Lee: None; Roberto Ruiz-Cordero: None; Annemieke van Zante: None

Background: Determining the origin of poorly differentiated carcinomas in the head and neck (H\&N) can be challenging but is critical for appropriate clinical management and informs prognosis. To address this issue, we examined the utility of next-generation sequencing (NGS) of poorly differentiated carcinomas of the H\&N. The impact of molecular testing on diagnosis, prognosis, and treatment was recorded.

Design: Surgical and/or cytologic specimens from patients with poorly differentiated carcinomas of the H\&N, including many with squamous differentiation, were selected for NGS with a 500+ gene panel. These tumors were either difficult to classify or of unknown origin based on morphology and immunohistochemistry alone (Table 1).

Results: Sequencing was performed on $12 \mathrm{H} \& \mathrm{~N}$ carcinomas. Seven cases of squamous cell carcinoma (SCC) were included. Two of the SCCs had known primaries (tonsil and hypopharynx), whereas the remainder $(n=5)$ were of unknown origin at presentation. Among these seven cases, NGS distinguished cutaneous $(n=3)$ from noncutaneous $(n=4)$ origin by the presence of UV mutational signature along with specific mutations common in cutaneous tumors. Five additional cases with squamous differentiation were tested. These included 3 anaplastic thyroid carcinomas and 2 salivary gland carcinomas; these 5 cases showed mutational profiles that are uncommon 
in SCC of the H\&N (Table 1). In many of the cases, the molecular findings had diagnostic, prognostic, and/or therapeutic implications. For example, the presence of a PTCH1 mutation coupled with UV signature supported the diagnosis of basal cell carcinoma metastatic to the lung in one patient. Mutation in PTEN with amplification of JAK2 and CD274 was supportive of SCC of lung origin in another patient. The amplification of 11q13.3 in one case has been associated with poor prognosis in HPV-negative SCC. One non-cutaneous SCC that was positive for p16 by immunohistochemistry and high-risk HPV by in situ hybridization showed a PIK3CA mutation, which can be a therapeutic target. TSC1 mutations are associated with everolimus response, making two of the patients potential candidates for this drug. EML4-ALK fusion was identified in a case of salivary duct carcinoma and $B R A F$ V600E mutation was identified in a case of anaplastic thyroid carcinoma, supporting targeted therapy for these patients.

Table 1. Clinicopathologic features of head and neck carcinomas.

\begin{tabular}{|c|c|c|c|c|c|c|c|c|}
\hline Case & Diagnosis & $\begin{array}{l}1^{\circ} \text { Tumor } \\
\text { location }\end{array}$ & $\begin{array}{l}\text { Distant } \\
\text { mets }\end{array}$ & $\begin{array}{l}\text { Ancillary } \\
\text { tests }\end{array}$ & Genes mutated & $\begin{array}{l}\text { UV } \\
\text { signature }\end{array}$ & Tx & $\begin{array}{l}\text { Pt } \\
\text { survival } \\
\text { (mo) }\end{array}$ \\
\hline 1 & SCC & $\begin{array}{l}\text { Potential } \\
\text { parotid }\end{array}$ & $\begin{array}{l}\text { Lungs, } \\
\text { liver, } \\
\text { bone, ST }\end{array}$ & & PTEN, TSC1, TP53 & Absent & CR & AWD, 12 \\
\hline 2 & SCC & $\begin{array}{l}\text { Probable } \\
\text { skin (ear) }\end{array}$ & 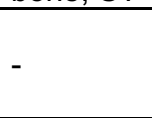 & p16- & $\begin{array}{l}\text { AR, CDKN2A, } \\
\text { NOTCH1, TP53, } \\
\text { TSC1, FAT1 }\end{array}$ & Present & IT & $\begin{array}{l}\text { AWOD, } \\
29\end{array}$ \\
\hline 3 & SCC & Lingual tonsil & - & $\begin{array}{l}\text { p16+, } \\
\text { HPV } \\
\text { ISH+ }\end{array}$ & PIK3CA & Absent & SR & $\begin{array}{l}\text { AWOD, } \\
11\end{array}$ \\
\hline 4 & $\begin{array}{l}\text { Salivary } \\
\text { duct ca }\end{array}$ & Parotid & - & & $\begin{array}{l}\text { EML4-ALK fusion, } \\
\text { CDKN2A, PTEN, } \\
\text { TP53 }\end{array}$ & Absent & SR & $\begin{array}{l}\text { AWOD, } \\
10\end{array}$ \\
\hline 5 & SCC & $\begin{array}{l}\text { Unknown- } \\
\text { potential lung }\end{array}$ & - & p16- & $\begin{array}{l}\text { TP53, CYLD, } \\
\text { CCND1 amp }\end{array}$ & Absent & $\begin{array}{l}\mathrm{SR}, \\
\mathrm{CR}\end{array}$ & $\begin{array}{l}\text { AWOD, } \\
9\end{array}$ \\
\hline 6 & SCC & Skin & $\begin{array}{l}\text { Skin to } \\
\text { Parotid }\end{array}$ & & $\begin{array}{l}\text { CDKN2A, NOTCH1, } \\
\text { TERT, TP53 }\end{array}$ & Present & $\begin{array}{l}\text { SR, } \\
\mathrm{R}\end{array}$ & AUD, 5 \\
\hline 7 & $\mathrm{BCC}$ & Lip & Lungs & & $\begin{array}{l}\text { ATRX, CIC, KMT2D, } \\
\text { MEN1, PPM1D, } \\
\text { PTCH1, TERT, TP53, } \\
\text { EPHA3 }\end{array}$ & Present & $\begin{array}{l}\mathrm{SR}, \\
\mathrm{C}\end{array}$ & AWD, 63 \\
\hline 8 & SCC & $\begin{array}{l}\text { Probable } \\
\text { hypopharynx }\end{array}$ & Lungs & p16- & $\begin{array}{l}\text { TP53; CDKN2A/B, } \\
\text { LRP1B loss; CCND1, } \\
\text { FGF19, FGF4 amp }\end{array}$ & Absent & IT & AWD, 15 \\
\hline 9 & $\begin{array}{l}\text { Anaplastic } \\
\text { thyroid ca }\end{array}$ & Thyroid & $\begin{array}{l}\text { Lung, } \\
\text { bone, } \\
\text { brain }\end{array}$ & & $B R A F, T E R T$ & Absent & C & DOD, 5 \\
\hline 10 & SCC & Hypopharynx & Parotid & & $\begin{array}{l}\text { TP53, ARID2, IGF2R, } \\
\text { NFE2L2; } \\
\text { CDKN2A/B loss; } \\
\text { MDM2, TERT amp }\end{array}$ & Absent & $\mathrm{R}$ & $\begin{array}{l}\text { AWOD, } \\
60\end{array}$ \\
\hline 11 & $\begin{array}{l}\text { Anaplastic } \\
\text { thyroid ca }\end{array}$ & Thyroid & $\begin{array}{l}\text { Lungs, } \\
\text { cervical } \\
\text { spine }\end{array}$ & & $\begin{array}{l}\text { PTEN; MDM2, JAK2, } \\
\text { CD274, } \\
\text { PDCD1LG2 amp; } \\
\text { TERT reargmnt }\end{array}$ & Absent & $\begin{array}{l}\text { R, } \\
\text { IT }\end{array}$ & AWD, 7 \\
\hline 12 & $\begin{array}{l}\text { Favor } \\
\text { anaplastic } \\
\text { thyroid ca }\end{array}$ & Thyroid & Lungs & & $B R A F, T E R T$ & Absent & CR & DOD, 2 \\
\hline
\end{tabular}

SCC, squamous cell carcinoma; LN, lymph node; ST, soft tissue; mets, metastases; HPV, high-risk human papillomavirus; ISH, in situ hybridization; amp, amplification; reargmnt, rearrangement; Tx, treatment; CR, chemoradiation; IT, immunotherapy; SR, surgical resection; R, radiation therapy; C, chemotherapy; mo, months; DOD, dead of disease; AWOD, alive without disease; AWD, alive with disease; AUD, alive with unknown disease progression 
Conclusions: NGS aids in accurately classifying carcinomas of the H\&N when histomorphology and immunohistochemistry are inconclusive. Our case series supports the utility of NGS in distinguishing cutaneous SCC vs. SCC of mucosal origin vs. carcinomas with squamous differentiation arising at other sites. NGS can additionally provide prognostic information and support the rational selection of targeted therapies.

\section{SWI/SNF Alterations in Poorly-Differentiated Sinonasal Carcinomas: An Institutional Experience Identifying INI1- and BRG1-Deficient Carcinomas via Immunohistochemistry Anne McLean-Holden ${ }^{1}$, Oluwaseun Ogunbona ${ }^{2}$, Daniel Lubin ${ }^{1}$, Kelly Magliocca ${ }^{3}$, Zaid Mahdi ${ }^{1}$, Faisal Saeed ${ }^{1}$, Qiuying Shi ${ }^{3}$ \\ ${ }^{1}$ Emory University Hospital, Atlanta, GA, ${ }^{2}$ Emory University School of Medicine, Atlanta, GA, ${ }^{3}$ Emory University, Atlanta, GA}

Disclosures: Anne McLean-Holden: None; Oluwaseun Ogunbona: None; Daniel Lubin: None; Kelly Magliocca: None; Zaid Mahdi: None; Faisal Saeed: None; Qiuying Shi: None

Background: Categorization of poorly-differentiated carcinomas of the sinonasal tract remains a challenge in head and neck pathology today. Within the last 10 years, significant discoveries regarding genetic distinction of tumors previously diagnosed as "sinonasal undifferentiated carcinoma" (SNUC) have allowed for improved classification of these challenging lesions. Inactivation of the SWItch/Sucrose Non-Fermentable (SWI/SNF) subfamily of ATPdependent chromatin remodeling complexes, including INI1 (SMARCB1) and BRG1 (SMARCA4), has been demonstrated in several case series investigating poorly-differentiated sinonasal carcinomas. Here, we report a series of INI1- and BRG1-deficient sinonasal carcinomas identified at our institution, with an emphasis on their morphologic and immunohistochemical features.

Design: The archives of Emory University Hospital were searched for cases of poorly differentiated sinonasal carcinoma. INI1 and BRG1 immunohistochemistry was performed, and cases were characterized clinically and microscopically.

Results: Six cases of INI1-deficient carcinoma and 2 cases of BRG1-deficient carcinoma were identified. Patients with INI1-deficient carcinoma ranged in age from 21 to 84 years, and 5 patients were male. Histologically, 2 INI1deficient carcinomas exhibited predominantly basaloid cytomorphology; 3 cases had mostly epithelioid tumor cells; and one case showed focal rhabdoid features. Only two cases showed high-grade features (increased atypia, necrosis and mitoses). All cases were positive for AE1/AE3, and showed loss of INI-1. Five cases were positive for p40, p63, or both; and just 1 case was positive for synaptophysin. Patients with BRG1-deficient carcinoma were both male, ages 53 and 57 years. One case was predominantly basaloid, with focal epithelioid cells and glandular formation. The other was epithelioid with focal rhabdoid features. Both BRG1-deficient cases showed high-grade traits. Both tumors were positive for AE1/AE3 and synaptophysin, and demonstrated loss of BRG-1. One case was weakly and focally positive for p40.

\begin{tabular}{|c|c|c|c|c|c|c|c|c|c|c|}
\hline $\begin{array}{l}\text { Case } \\
\#\end{array}$ & $\begin{array}{l}\text { Tumor } \\
\text { size, } \\
\text { cm }\end{array}$ & BRG-1 & INI-1 & AE1/3 & Synapto-physin & $\mathrm{p} 40$ & p63 & NUT-1 & $\begin{array}{l}\text { SOX10 } \\
\text { /S100 }\end{array}$ & CD99 \\
\hline 1 & 3.7 & NR & Lost & + & - & NR & - & NR & $-/-$ & NR \\
\hline 2 & 6.5 & NR & Lost & + & - & + & NR & - & -1 & NR \\
\hline 3 & 4.2 & NR & Lost & + & - & + focal & + focal & - & -1 & NR \\
\hline 4 & 3.5 & NR & Lost & + & - & NR & + & - & $1-$ & - \\
\hline 5 & 9.1 & NR & Lost & + & - & + & + & - & $-/-$ & NR \\
\hline 6 & 4.9 & NR & Lost & + & + focal & + & NR & - & $1-$ & NR \\
\hline 7 & 4.9 & Lost & Retained & + & + & - & - & - & - & - \\
\hline 8 & 4.1 & Lost & Retained & + & + & + focal & NR & NR & $\begin{array}{l}+/+ \\
\text { isolated }\end{array}$ & - \\
\hline
\end{tabular}


Figure $1-653$

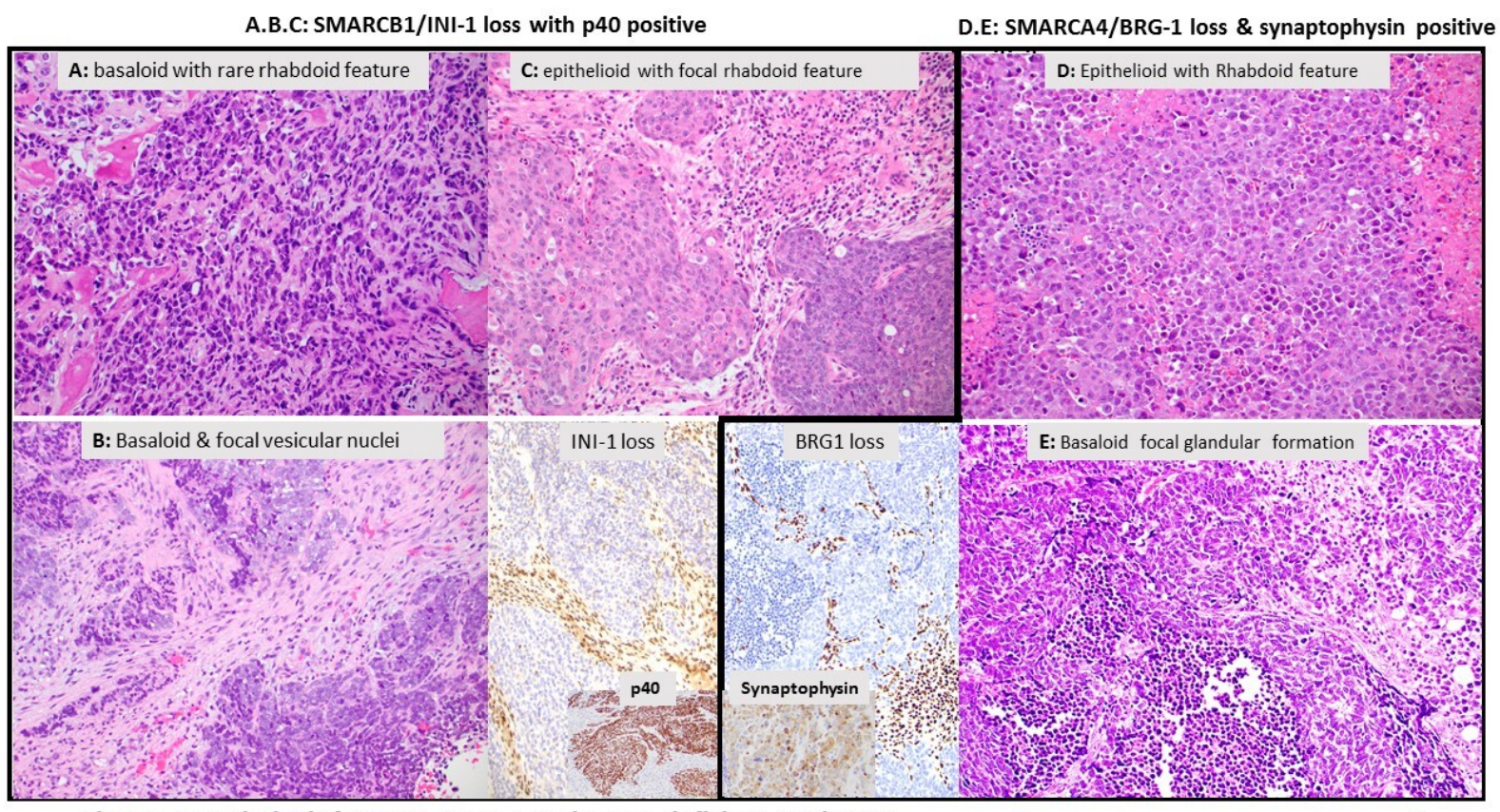

Figure: Morphologic features on INI-1 and BRG-1 deficient carcinoma

(A. Case 1; B. Case 2; C. Case 3; D. Case 7; E. Case 8)

Conclusions: SWI/SNF alterations in poorly-differentiated carcinomas of the sinonasal tract are well-described. We show that INI1-deficient carcinomas may exhibit lower-grade morphology than BRG1-deficient cases. Cytomorphology is not an accurate predictor of loss of either BRG-1 or INI-1. Tumors that appear neuroendocrine in nature may show loss of either marker, representing a potential diagnostic pitfall. Increased awareness of these entities allows for their diagnostic consideration when confronted with a poorly-differentiated tumor of the sinonasal tract.

\section{Signet-Ring Cell Change in Adenoid Cystic Carcinoma: An Institutional Experience Anne McLean-Holden ${ }^{1}$, Daniel Lubin ${ }^{1}$, Qiuying Shi ${ }^{2}$, Faisal Saeed ${ }^{1}$, Zaid Mahdi ${ }^{1}$, Kelly Magliocca ${ }^{2}$ ${ }^{1}$ Emory University Hospital, Atlanta, GA, ${ }^{2}$ Emory University, Atlanta, GA}

Disclosures: Anne McLean-Holden: None; Daniel Lubin: None; Qiuying Shi: None; Faisal Saeed: None; Zaid Mahdi: None; Kelly Magliocca: None

Background: Background. Signet-ring cell change (SRCC) occurring in adenoid cystic carcinoma (AdCC) has been previously described in a single study consisting of four female patients. As such, SRCC is currently not well characterized in AdCC and encountering this morphologic variant may pose diagnostic challenges, particularly in the small biopsy material. Here, we report a single institutional series of AdCC identified as having SRCC change with a focus on morphologic findings and clinical follow-up.

Design: Methods. The archives of Emory University Hospital were searched for patients with a diagnosis of adenoid cystic carcinoma affecting the upper aerodigestive tract between 1/1/2005 and 10/25/2020. Slides were retrieved and re-reviewed for SRCC, and further characterized clinically. Case consultations, ie: slide material only, in which the patient was not clinically evaluated, were excluded. Cases of cutaneous and breast AdCC were excluded.

Results: Results. Seventy-five patients with AdCC were evaluated at Emory University during the study interval. Microscopic slides for 65 cases were available for review. Nine cases of AdCC with SRCC were identified (14\%). Patients ranged in age from 29 to 73 years, and 7 of 9 patients were male. Histologically, all cases appeared biphasic with epithelial cells and myoepithelial cells. The predominant pattern of growth was tubular or tubularcribriform in 8 of 9 cases. Areas of solid growth, micropapillary formation and/or high grade transformation was 
identified in five cases. The presence of SRCC varied and ranged from a minor component $(<5 \%)$ to present in $50 \%$ or more of tumor. All cases showed perineural and intraneural invasion. Clinical outcome did not appear dissimilar to the known natural history of AdCC.

Conclusions: Conclusion. SRCC in adenoid cystic carcinoma is uncommon and in view of the unpredictable clinical course of AdCC, the morphologic finding remains of uncertain significance. Our results indicate that SRCC in AdCC tends to occur in male patients, within the maxillary/sinonasal region and may be more commonly identified than previously known. Finding SRCC within AdCC may additionally have value in distinguishing AdCC from tumors that appear microscopically similar but with a distinct clinical course, such as HPV-related multiphenotypic sinonasal carcinoma in which SRCC has not been described.

\section{SMARCB1(INI-1)-Deficient Head And Neck Carcinomas In Adults-A Tertiary Care Cancer Center Series of 17 Cases \\ Neha Mittal ${ }^{1}$, Munita Bal ${ }^{2}$, Swapnil Rane ${ }^{2}$, Asawari Patil ${ }^{3}$ \\ ${ }^{1}$ Tata Memorial Hospital, Mumbai, India, ${ }^{2}$ Tata Memorial Centre, Mumbai, India, ${ }^{3}$ Tata Memorial Centre, Thane West, India}

Disclosures: Neha Mittal: None; Munita Bal: None; Swapnil Rane: None; Asawari Patil: None

Background: A defect in the ubiquitously expressed genes of the SWI/SNF chromatin remodelling complex results in the SMARCB1(INI-1) deficient family of tumours which include Epithelioid sarcoma, Malignant rhabdoid tumours and recently defined SMARCB1 deficient sinonasal carcinoma (SDSC).

Design: A detailed retrospective review of clinical and histological parameters of SMARCB1/INI-1 deficient head and neck carcinomas in adults diagnosed from 2010 to date was undertaken.

Results: A total of 17 cases (13 males, 5 females with a M:F ratio of 2.6:1) of SMARCB1 deficient tumours comprising of 16 cases of SDSC and 1 case of SMARCB1 deficient myoepithelial carcinoma were evaluated. The mean age of the patients was 48.4 years (range 30-74 years); diminished vision and nasal bleed being the most common symptoms. The epicenter of the SDSCs was Orbital and maxillo-ethmoid region in $85 \%$ cases with intracranial extension in $50 \%$ of cases. The predominant histology was rhabdoid in $33.3 \%$, basaloid in $56.25 \%$; mixed in $6.25 \%$, and eosinophilic subtype in $6.25 \%$ cases. Glandular differentiation was seen in 2 cases(12.5\%), scattered rhabdoid cells, and microcystic change/intracytoplasmic vacuolations in $72.2 \%$ and stromal myxoid change in $83.3 \%$ cases. On immunohistochemistry, diffuse loss of INI-1characterized all the cases along with variable positivity for keratin, p40 in 50\%, and p16 and synaptophysin in $6.25 \%$ cases each. Metastasis at presentation was seen in 9 cases; nodal in 6, and distant in 3 cases. Trimodality therapy with curative intent was administered in 8 patients, 3 of whom recurred within 6 months. At a mean follow-up of 1 year, only 2 patients were alive without disease.

\begin{tabular}{|l|l|}
\hline Parameter & Results $\mathbf{( n = 1 6 )}$ \\
\hline Epidemiology & $30-74$ years \\
\hline Age range & $2.6: 1(13$ males, 5 females) \\
\hline M:F & 8 cases \\
\hline Site/Extent of tumour & 7 cases \\
\hline Maxillo-ethmoid & 1 case \\
\hline Ethmoid & 8 cases \\
\hline Sphenoid & 8 cases \\
\hline Orbital involvement & \\
\hline Intracranial extension & 5 cases \\
\hline Symptoms & 8 cases \\
\hline Nasal bleed & 3 cases \\
\hline Diminished vision & 2 cases \\
\hline Proptosis & \\
\hline Nasal obstruction & \\
\hline
\end{tabular}




\begin{tabular}{|c|c|}
\hline \multicolumn{2}{|l|}{ Histology } \\
\hline Basaloid & 9 cases \\
\hline Rhabdoid & 5 cases \\
\hline Mixed basaloid and rhabdoid & 1 case \\
\hline "Pink tumour appearance" & 1 case \\
\hline Scattered rhabdoid cells & 13 cases $(5 \%-30 \%)$ \\
\hline Microcystic/ intracytoplasmic vacuolation & 13 cases \\
\hline Glandular differentiation & 2 cases \\
\hline Clear cells & 4 cases \\
\hline Squamous differentiation (keratin) & None \\
\hline Stromal mucin & 14 cases \\
\hline Mitosis >10/10HPF & 14 cases \\
\hline Necrosis & 6 cases \\
\hline \multicolumn{2}{|l|}{ Immunohistochemistry } \\
\hline INI-1 loss & All cases \\
\hline Keratin positive & All cases \\
\hline P63/p40 positive & 8 cases \\
\hline S100 & Negative in all cases $(n=10)$ \\
\hline Synaptophysin positive & 1 case $(n=10)$ \\
\hline NUT & Negative $(n=5)$ \\
\hline P16 positive (>70\% nuclear and cytoplasmic) & 1 case $(n=8)$ \\
\hline \multicolumn{2}{|l|}{ Treatment } \\
\hline Surgery+CTRT & 8 cases \\
\hline Palliative treatment & 5 cases \\
\hline CTRT & 1 case \\
\hline No details of treatment & 2 cases \\
\hline \multicolumn{2}{|l|}{ Follow up } \\
\hline Nodal metastasis at presentation & 6cases \\
\hline Distant metastasis at presentation & 3cases \\
\hline Recurrence & 3 cases \\
\hline Not known & 2 cases \\
\hline Alive without disease & 2 cases \\
\hline Alive with disease & 3 case \\
\hline Dead of disease & 9 cases \\
\hline Not known & 2 cases \\
\hline
\end{tabular}

Conclusions: Elderly male, orbital and maxillo-ethmoid involvement, rhabdoid cells, and INI-1 loss characterize SDSCs. Unique histology, an aggressive clinical course, and an identifiable driver mutation necessitate accurate identification for prognostication and prospects of targeted therapy in the near future.

\section{Wide Spectrum of Lesions Arising in the External Auditory Canal: Case-Series at a Single Tertiary-Care Oncology Center \\ Manando Nakasaki ${ }^{1}$, Cary Johnson ${ }^{1}$, Harrison Lin ${ }^{2}$, Hamid Djalilian ${ }^{3}$, Edward Kuoy ${ }^{4}$, Beverly Wang ${ }^{3}$ \\ ${ }^{1}$ University of California, Irvine, Irvine, CA, ${ }^{2}$ UC Irvine Medical Center, Irvine, CA, ${ }^{3}$ UCI Medical Center, Orange, CA, ${ }^{4}$ UC Irvine Medical Center, Orange, $C A$}

Disclosures: Manando Nakasaki: None; Cary Johnson: None; Harrison Lin: None; Hamid Djalilian: None; Edward Kuoy: None; Beverly Wang: None

Background: Although the external auditory canal (EAC) is a short, narrow chamber, it is often the site of a wide spectrum of lesions, partly because the EAC is composed of various types of tissues including cutaneous, glandular, bone, and cartilage components. We aimed to investigate the prevalence and spectrum of neoplasms and tumor-like lesions to study their clinical and pathologic features.

Design: Retrospective review of the clinicopathologic features of both primary neoplastic and tumor-like lesions of EAC diagnosed from 2013-2020. 
Results: A total of 43 cases were included, 25 males and 18 females, median age 58 years (range, 7-89). Nonspecific inflammatory and infectious lesions excluded. Twenty six cases were benign tumor/tumor-like lesions and 17 were malignant tumors. Thirty three were cutaneous in origin, 7 were derived from bone and soft tissue origin and 3 were hematopoietic in origin. Eighty seven percent the majority of the malignant tumors were cutaneous in origin and consisted of 10 squamous cell carcinomas, 3 basal cell carcinomas, 1 mucoepidermoid carcinoma, and 1 sebaceous carcinoma. Two of the three hematopoietic cases were malignant (CD30 positive T cell lymphoma and myeloid sarcoma). Hearing change, otalgia and tinnitus were seen in $13(41 \%), 4(13 \%)$ and 1 $(3 \%)$ cases respectively.

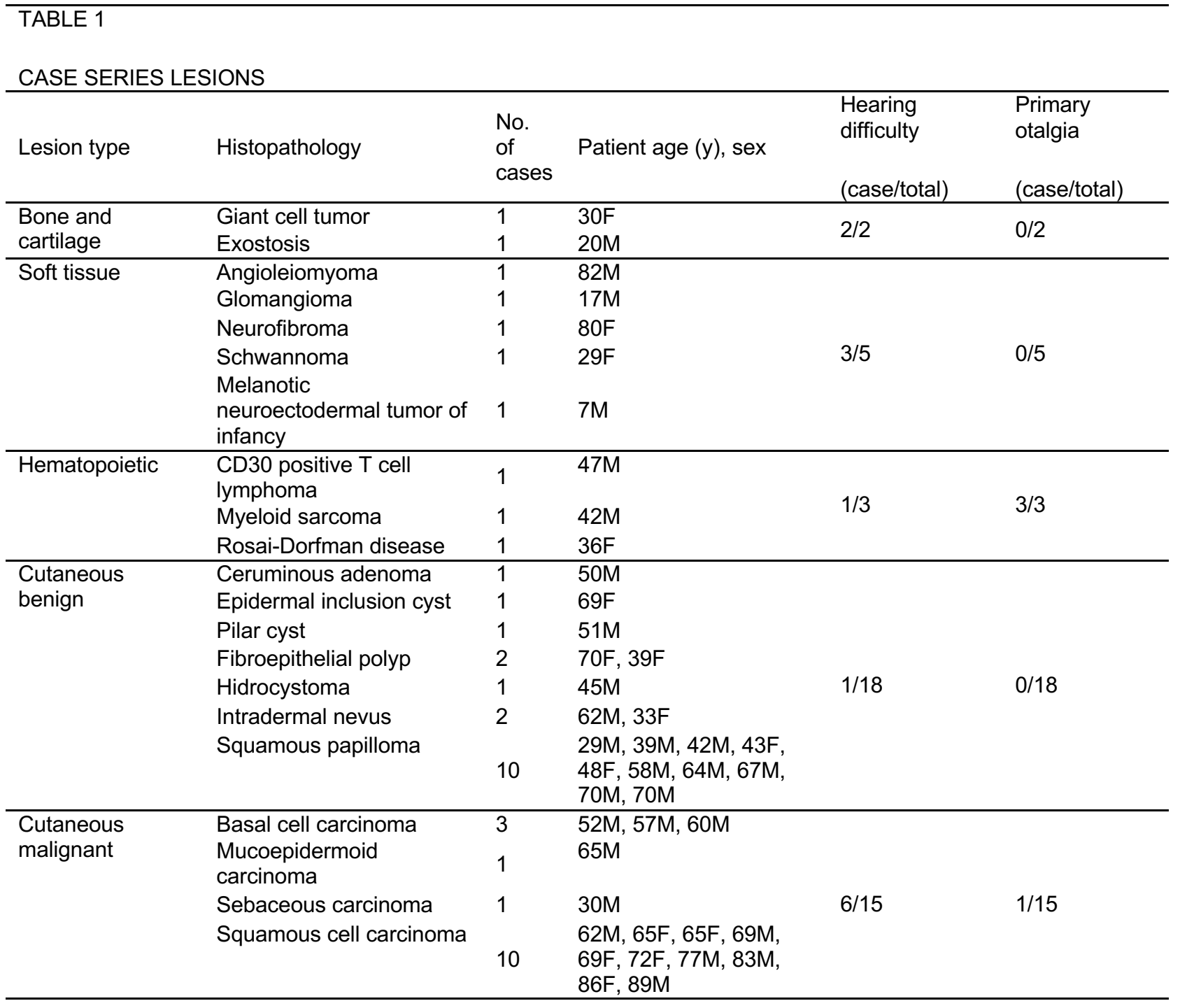

Conclusions: Although tumor/tumor-like lesions are relatively rare in the EAC, they show a wide variety of histopathologic origins. The most common malignant and benign tumors being squamous cell carcinoma and squamous papilloma presenting predominantly as hearing change and as an incidental finding respectively. Interestingly all 3 hematopoietic neoplasms presented with primary otalgia. 


\section{Transcriptionally-Active High Risk HPV is a Frequent Driver of Orbital and Periocular Squamous Cell Carcinomas \\ James Nix ${ }^{1}$, Nyall London ${ }^{1}$, Carole Fakhry ${ }^{1}$, Charles Eberhart ${ }^{2}$, Lisa Rooper ${ }^{1}$ \\ ${ }^{1}$ Johns Hopkins Hospital, Baltimore, MD, ${ }^{2}$ Johns Hopkins University School of Medicine, Baltimore, MD}

Disclosures: James Nix: None; Nyall London: None; Carole Fakhry: None; Charles Eberhart: None; Lisa Rooper: None

Background: Squamous cell carcinomas (SCC) involving the orbit and periocular regions can arise from a wide range of sites, including the caruncle, lacrimal sac and ducts, lacrimal gland, conjunctiva, and eyelid skin. The pathogenesis of those orbital SCC that lack a clear cutaneous or conjunctival origin is poorly understood. In particular, there is conflicting evidence regarding the role of human papillomavirus (HPV) in SCC in this area. While some studies have reported HPV involvement in up to $90 \%$ of orbital SCC by p16 immunohistochemistry (IHC), DNA in situ hybridization (ISH), or polymerase chain reaction (PCR), others have failed to identify HPV in these tumors. This study applies the gold standard HPV RNA ISH to clarify the role of transcriptionally active HPV in orbital SCC.

Design: All orbital and periocular SCC were identified from the surgical pathology archives of a large academic medical center between 1996 and 2019. All available clinical and radiographic data was reviewed, and those extending from a conjunctival, cutaneous, or sinonasal primary were excluded. A tissue microarray was constructed to include 9 tumors; 2 other tumors were evaluated on whole slide sections. Immunohistochemistry for p16 and RNA ISH for high-risk HPV (using a cocktail probe recognizing 18 types) were performed on all cases. Typespecific RNA ISH for HPV 16 was performed on a subset of cases as tissue availability permitted.

Results: 11 cases of orbital and periocular SCC were identified in 7 men and 4 women with a median age of 73 years. Tumors arose in the lacrimal sac/nasolacrimal duct $(n=8)$, lacrimal gland $(n=2)$, and in the soft tissue of an anophthalmic socket $(n=1)$. Overall 8/11 tumors $(73 \%)$ were positive for $p 16$ and $7 / 11(64 \%)$ were positive for highrisk HPV; p16 IHC was $100 \%$ sensitive and $88 \%$ specific for HPV involvement. The HPV-positive tumors included $5 / 8$ in the lacrimal sac/nasolacrimal duct $(63 \%)$ and $2 / 2$ in the lacrimal gland (100\%); the tumor arising in the anophthalmic socket was HPV negative. All 6 cases with tissue available for testing were positive for HPV 16.

Conclusions: Evaluation with RNA ISH demonstrates that transcriptionally active high-risk HPV, particularly HPV type 16, is a driver of a majority of orbital and periocular SCC. These results confirm previous DNA ISH and PCRbased reports that HPV is extremely common in lacrimal sac/nasolacrimal duct SCC, raising the question of whether these tumors share overlapping pathogenesis with the HPV hotspot in the sinonasal tract. They also highlight the lacrimal gland as a rare and novel site of HPV involvement. HPV testing for tumors in orbital and periocular sites may open the door to emerging HPV-targeted treatment and monitoring strategies in patients with advanced disease.

\section{Comprehensive Molecular Profiling of Sinonasal Teratocarcinosarcoma Identifies Recurrent SMARCA4 and Wnt/ß-catenin Pathway Mutations Lisa Rooper ${ }^{1}$, Nasir Ud Din², Jeffrey Gagan ${ }^{3}$, Lester Thompson ${ }^{4}$, Abbas Agaimy ${ }^{5}$, Justin Bishop ${ }^{3}$ ${ }^{1}$ Johns Hopkins Hospital, Baltimore, MD, ${ }^{2}$ The Aga Khan University Hospital, Karachi, Pakistan, ${ }^{3}$ UTSouthwestern Medical Center, Dallas, TX, ${ }^{4}$ Southern California Permanente Medical Group, Woodland Hills, CA, ${ }^{5}$ Universitätsklinikum, Erlangen, Germany}

Disclosures: Lisa Rooper: None; Nasir Ud Din: None; Jeffrey Gagan: None; Lester Thompson: None; Abbas Agaimy: None; Justin Bishop: None

Background: Sinonasal teratocarcinosarcoma (TCS) is a rare and aggressive sinonasal tumor defined by intermixed malignant epithelial, mesenchymal, and primitive neuroepithelial components. While this divergent differentiation has historically engendered controversy about its histogenesis, recent evidence shows that most TCS have SMARCA4 loss by immunohistochemistry (IHC), suggesting these tumors may be more closely related to sinonasal carcinomas. However the molecular underpinnings of TCS, particularly those cases with partial or intact SMARCA4 IHC expression, have not been fully characterized. In this study, we performed next generation 
sequencing (NGS) on a series of TCS with known SMARCA4 IHC status to better evaluate the genetic findings in these tumors and their implications for classification.

Design: 8 cases of sinonasal TCS from 2 large academic medical centers and the authors' consultation files were reviewed, including 4 cases previously shown to have complete loss of SMARCA4 by IHC, 2 with partial SMARCA4 expression, and 2 with intact SMARCA4. NGS was performed on the Illumina NextSeq 550 using a custom panel including all exons from >1425 cancer-related genes. Based on molecular results, $ß$-catenin IHC was performed on all cases with sufficient tissue available.

Results: 5 cases (63\%) showed biallelic inactivation of SMARCA4, including 4 cases with complete SMARCA4 loss by IHC and 1 case with partial expression. 3 cases (38\%) demonstrated Wnt/ß-catenin pathway mutations, including 2 with CTNNB1 mutations that showed partial SMARCA4 expression by IHC and 1 with APC mutation and intact SMARCA4 IHC. 1 of these cases demonstrated both SMARCA4 inactivation and CTNNB1 mutation. 1 additional case (13\%) lacked SMARCA4 or Wnt/ß-catenin pathway mutations and instead demonstrated MUTYH mutation and biallelic copy loss of RB1 and MAP3K1. 6 of 7 cases tested (86\%) showed focal nuclear ß-catenin expression, including cases with SMARCA4 inactivation and Wnt/ß-catenin mutations.

Conclusions: Inactivation of SMARCA4 appears to be the most common genetic event in sinonasal TCS and generally leads to complete loss of SMARCA4 by IHC. However, activating Wnt/ß-catenin pathway mutations are also seen in a significant subset of tumors, with CTNNB1 mutations also associated with partial SMARCA4 protein expression. These findings are not mutually exclusive, and nuclear $ß$-catenin can be identified in both groups. This overlap in pathogenesis and protein expression may stem from established interactions between SMARCA4 and the $\mathrm{Wnt} / \mathrm{ß}$-catenin pathway. Both of these molecular events have previously been reported in sinonasal carcinomas with neuroendocrine differentiation, further supporting a relationship between TCS and sinonasal carcinomas.

\section{MYB Expression by RNA In-Situ Hybridization is Sensitive and Specific for Adenoid Cystic Carcinoma \\ Xiaoyu Tang ${ }^{1}$, Jianhui Shi ${ }^{1}$, Fan Lin ${ }^{1}$, Rob Monroe ${ }^{2}$, Haiyan Liu ${ }^{1}$ \\ ${ }^{1}$ Geisinger Medical Center, Danville, PA, ${ }^{2}$ Advanced Cell Diagnostics, Inc, Newark, CA}

Disclosures: Xiaoyu Tang: None; Jianhui Shi: None; Fan Lin: None; Rob Monroe: Employee, Bio-Techne; Haiyan Liu: None

Background: Diagnosis of adenoid cystic carcinoma (AdCC) on fine-needle aspiration (FNA) specimen can be challenging due to limited diagnostic material and overlapping cellular morphology with other basaloid salivary gland neoplasms. Immunohistochemistry $(\mathrm{IHC})$ assists in the differential diagnosis; however, most available antibodies have limitations in specificity and sensitivity. The recurrent $t(6 ; 9)(q 22-23 ; p 23-24)$ translocation in AdCC results in MYB-NFIB fusion, leading to overexpression of MYB protein and RNA, which was reported to be specific for AdCC. In the current study, we evaluated the expression of MYB by RNA in-situ hybridization (ISH) on cell blocks (CB) of cytology FNA of salivary gland basaloid neoplasms and on tissue microarrays (TMA) of surgical AdCCs, to assess its utility in distinguishing AdCC from mimics in cytology FNA samples.

Design: Seventeen cytology FNAs of salivary gland basaloid neoplasms with confirmed diagnosis on follow-up surgical resection and CBs containing lesional cells were retrieved, including $\operatorname{AdCC}(n=6)$, pleomorphic adenoma $(n=5)$, basal cell adenoma $(n=5)$, and lymphadenoma $(n=1)$. Also retrieved are 17 surgical AdCCs from 3 anatomic sites, including head and neck $(n=9)$, trachea and lung $(n=4)$, and breast $(n=4)$. TMA of the surgical AdCCs was constructed with one punch $(1 \mathrm{~mm})$ of each tumor.

MYB RNA ISH (MYB RNA probe, Advanced Cell Diagnostics) was performed on CBs of 17 cytology FNA samples and on TMA of 17 surgical AdCCs using the Leica Bond III automated staining platform. The staining distribution was recorded as negative ( $<2$ dots/tumor cell and in $<5 \%$ of tumor cells), $1+\left({ }^{3} 2\right.$ dots and in $\left.>5 \%\right), 2+$ (clustered signals in $5-49 \%$ of tumor cells); $3+$ (clustered signals in $50-75 \%$ ), $4+$ (clustered signals in $75-100 \%$ ).

Results: The MYB expression by RNA ISH on CBs of cytology FNA samples and on TMA of surgical AdCCs is summarized in Table 1. In cytology FNA samples, MYB expression was identified in $83 \%$ (5/6) of AdCCs (Fig.1), but none of the others (Fig.2) except the case of lymphadenoma (1+). MYB espression by RNA ISH had a 
sensitivity of $83 \%$ and a specificity of $91 \%$ for AdCC in cytology FNA samples. In surgical AdCCs on TMA, the sensitivities of MYB RNA ISH in 3 groups of AdCCs are 100\% (9/9) for head and neck, $50 \%(2 / 4)$ for trachea and lung, and $100 \%$ (4/4) for breast.

MYB expression by RNA ISH had an overall sensitivity of $87 \%(20 / 23)$ and specificity of $91 \%(1 / 11)$ for AdCCs. The surgical AdCCs from trachea and lung showed lower sensitivity $(50 \%$, compared with $100 \%$ for AdCCs from trachea/lung, and breast).

Table 1. Summary of MYB Expression by RNA ISH on Cell Blocks of Cytology FNAs and TMA of Surgical AdCCs

\begin{tabular}{|c|c|c|c|c|c|c|}
\hline \multirow{2}{*}{ Diagnosis/Organ } & \multirow[t]{2}{*}{ MYB Neg. } & \multicolumn{4}{|c|}{ MYB Pos. } & \multirow[t]{2}{*}{ Total Pos. N (\%) } \\
\hline & & $1+$ & $2+$ & $3+$ & 4+ & \\
\hline \multicolumn{7}{|l|}{ Cytology FNA on CB: } \\
\hline Adenoid cystic CA (n=6) & 1 & 1 & 1 & 1 & 2 & $5(83 \%)$ \\
\hline Pleomorphic adenoma $(n=5)$ & 5 & & & & & 0 \\
\hline Basal cell adenoma $(n=5)$ & 5 & & & & & 0 \\
\hline Lymphadenoma $(\mathrm{n}=1)$ & 0 & 1 & & & & $1(100 \%)$ \\
\hline \multicolumn{7}{|l|}{ Surgical AdCC on TMA: } \\
\hline Head and Neck $(n=9)$ & 0 & 1 & 3 & 1 & 4 & $9(100 \%)$ \\
\hline Trachea and Lung $(n=4)$ & 2 & 1 & 1 & & & $2(50 \%)$ \\
\hline Breast $(n=4)$ & 0 & 1 & 2 & & 1 & $4(100 \%)$ \\
\hline
\end{tabular}

Figure $1-659$

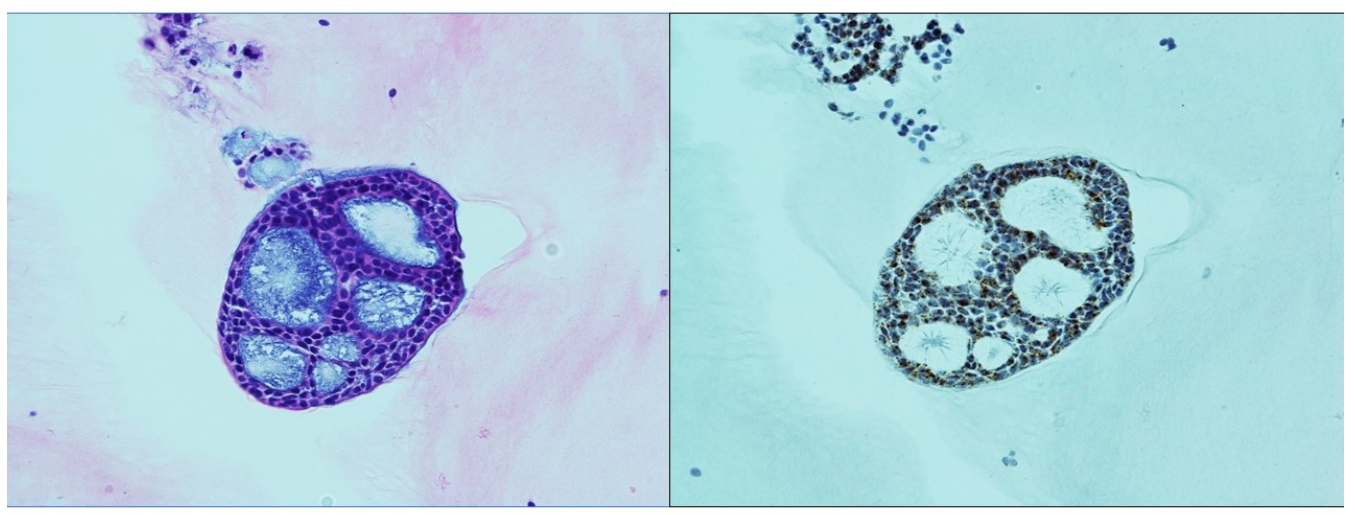

Fig. 1 H\&E and MYB RNA ISH on FNA cell block, a case of adenoid cystic carcinoma (x40) with 4+ positivity. H\&E, hematoxylin and eosin.

Figure 2 - 659

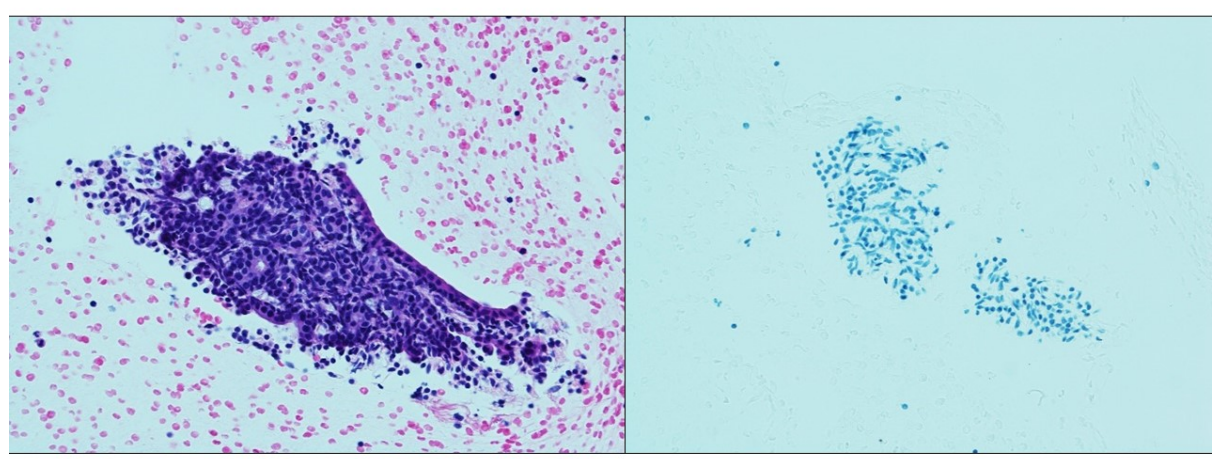

Fig. 2. H\&E and MYB RNA ISH on FNA cell block, a case of monomorphic adenoma (x40). H\&E, hematoxylin and eosin. 
Conclusions: Our data suggest that MYB expression by RNA ISH is sensitive and specific for AdCC, with an overall sensitivity of $87 \%$ and specificity of $91 \%$. MYB RNA ISH assay can be applied in assisting the diagnosis of AdCC on cytology FNA samples. The significance of lower sensitivity in AdCCs from trachea and lung is uncertain, requiring future large series of studies to validate or explore further of our findings

\section{Number of Foci of Perineural Invasion Predict Behavior in Low Stage Head and Neck Adenoid Cystic Carcinoma Lester Thompson, Southern California Permanente Medical Group, Woodland Hills, CA}

\section{Disclosures: Lester Thompson: None}

Background: Management of patients with low AJCC8 stage (Stage I and II) head and neck adenoid cystic carcinoma (HNACC) is unclear, with histologic perineural invasion (PNI) not yet critically assessed as an independent predictive factor in this cohort of patients.

Design: A retrospective review of surgically resected HNACCs was performed from 2000 to 2018 within Southern California Permanente Medical Group. Only patients with pathologic AJCC8 stage I or II disease after definitive surgery were included. All histologic material submitted was reviewed by standard H\&E stained slides. Factors determined included: presence or absence of PNI; number of foci of PNI, size of nerve with PNI (measured perpendicular to fiber direction and measured from $1 \mathrm{~mm}$ or greater); and distance of furtherest point of PNI from main primary tumor mass was recorded. No attempt was made to determine if the examined nerve was in continuity with other foci nearby, meaning one nerve may have more than 1 detectable are of PNI; foci were counted up to 100 foci as a maximum; perineural and intraneural invasion were not distinguished; PNI was defined by identifying neoplastic cells abutting the nerve, destroying the perineurium, and/or within the nerve substance; no minimum or maximum number of slides reviewed were required; no quantitation was attempted based on overall tumor size versus number of blocks submitted. Determined as a continuous variable, the counts were stratified into 3 groups: no perineural invasion; $\leq 15$ foci of PNI; and $>15$ foci of PNI. Analysis of PNI number which may predict for recurrence was performed using receiver operator curve analysis. No consideration was given to patient age, tumor site, tumor grade, tumor size, or margin status.

Results: Forty-six patients met inclusion criteria. On univariate analysis, locoregional recurrence was predicted by the number of PNI foci (HR 1.024, 95\% Cl 0.9992-1.049, $\mathrm{p}=0.03$ ), a finding that persisted on multivariate analysis (HR 1.024, 95\% Cl 1.00025-1.051, $\mathrm{p}=0.048$ ). Further, on multivariate analysis of predictors of any relapse, number of PNI foci was significant (HR 1.02981, 95\% Cl 1.00862-1.051, $\mathrm{p}=0.006)$. A receiver operator curve based on determining any disease recurrence as the outcome measure, yielded 17.5 foci of PNI. However, for ease of use, $\leq 15$ foci and $>15$ foci of PNI created dichotomous cohorts. Based on these groups, 5- and 10-year freedom from relapse were $96 \%$ versus $73 \%$ and $96 \%$ versus $47 \%$, respectively (HR 16.02, $p=0.0004$ ); 5 - and 10-year locoregional control were $96 \%$ versus $80 \%$ and $96 \%$ and $64 \%$ (HR 8.67, p=0.02); and 5- and 10-year distant control were $100 \%$ versus $80 \%$ and $100 \%$ versus $55 \%$ (HR $3.53 ; p<0.001)$. 


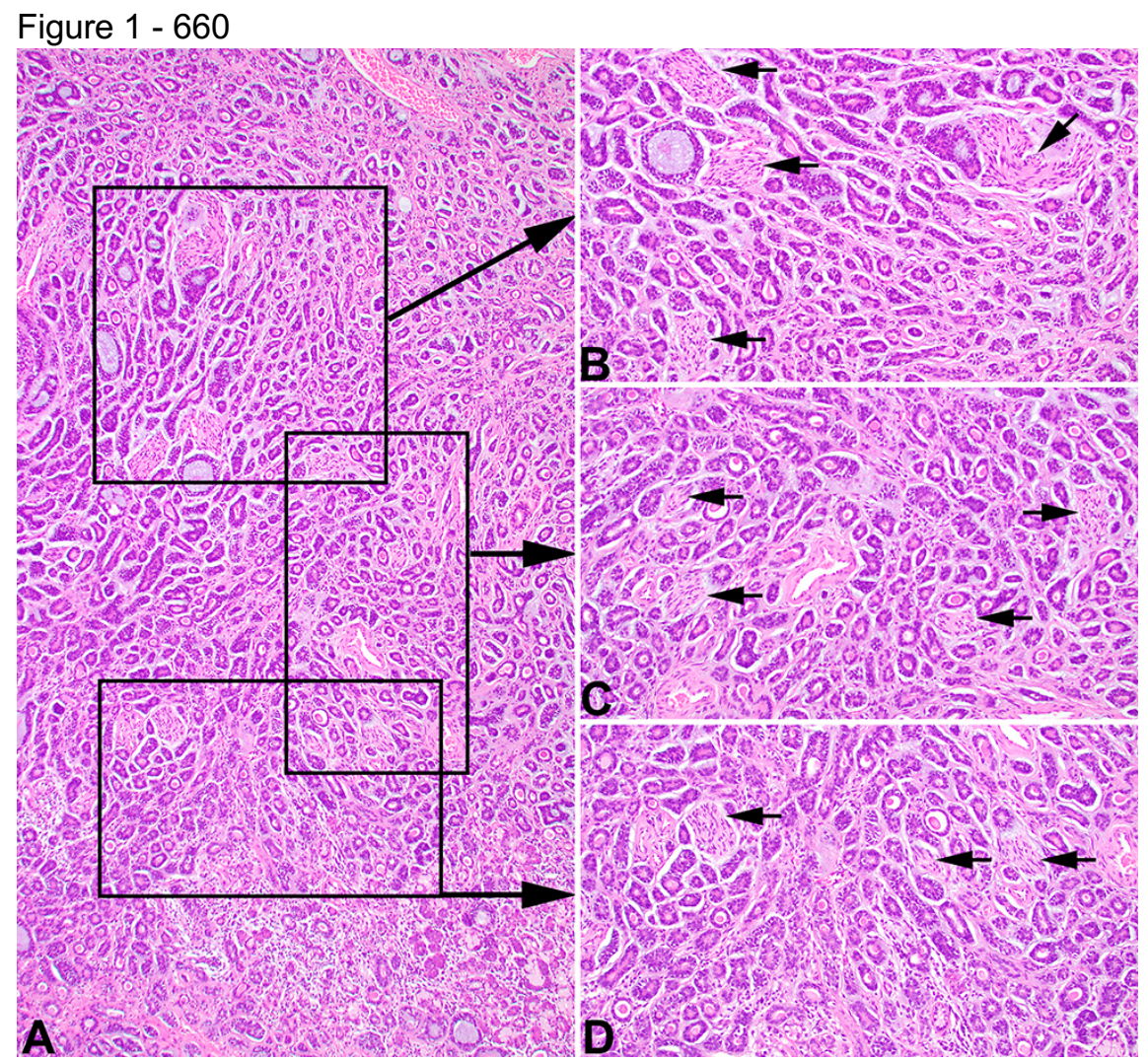

Conclusions: Total number of PNI foci, when divided into $\leq 15$ foci and $>15$ foci of PNI has been shown to be prognostic of any recurrence in early stage HNACC, unrelated to other variables. Thus, further evaluation may be considered when $>15$ foci of $\mathrm{PNI}$ are present.

\section{Parotid Gland Squamous Cell Carcinoma: Direct Cutaneous Invasion Versus Metastatic Disease: A Clinicopathologic Study of 95 Cases \\ Lester Thompson, Southern California Permanente Medical Group, Woodland Hills, CA}

\section{Disclosures: Lester Thompson: None}

Background: Squamous cell carcinoma (SCC) is the most common head and neck cancer, whether cutaneous (cSCC) or mucosal (mSCC), frequently metastasizing to regional lymph node basins or organs. Parotid gland SCC may represent metastatic SCC, direct invasion, or primary tumor, with separation sometimes difficult, including the differential with mucoepidermoid carcinoma (MEC). Management and outcome are quite different in these scenarios.

Design: A retrospective review of 95 patients with parotid gland SCC between 2011-2015 within Southern California Permanente Medical Group. Three categories were identified: 1) metastatic cSCC (McSCC); 2) direct invasion into the parotid gland from a primary cutaneous SCC (DcSCC); 3) metastatic SCC from a mucosal primary (mSCC). No primary salivary gland tumors were identified and mucocytes were not observed, excluding MEC. All except 12 patients were managed with radical excision and neck dissection; 63 received radiation therapy.

Results: Based on these 3 categories (Table):

51 patients with McSCC: Tumors arose from: forehead $(n=16)$; ear $(n=12)$; scalp $(n=8)$; cheek $(n=5)$; and 3 each for jawline, nose, and neck; eyelid $(n=1)$; and other skin sites $(n=4)$ a mean interval of 4.3 years before metastatic presentation. $65 \%$ of patients were alive without disease or dead from other causes (mean $5.3 \mathrm{yrs}$ ), while $35 \%$ were dead of disease (mean $1.8 \mathrm{yrs}$ ). 
41 patients with DcSCC: Tumors directly involved the parotid gland from an overlying cSCC. $49 \%$ of patients were alive without disease or dead from other causes (mean $5.6 \mathrm{yrs}$ ), while $49 \%$ were dead of disease (mean $1.1 \mathrm{yrs}$ ).

3 patients with OSCC. One tumor each from tongue, nasopharynx, and lung. Two patients, 1 dead and 1 alive, were without disease (average $3.7 \mathrm{yrs}$ ) and 1 dead of disease (1.8 yrs).

\section{Characteristics of Parotid Gland Squamous Cell Carcinoma}

\begin{tabular}{|c|c|c|c|c|}
\hline Clinical characteristics & $\begin{array}{l}\text { All cases } \\
(n=95)\end{array}$ & $\begin{array}{l}\text { Metastatic Cutaneous } \\
\text { SCC to Parotid Gland } \\
(n=51)\end{array}$ & $\begin{array}{l}\text { Cutaneous } \\
\text { SCC with } \\
\text { Direct Parotid } \\
\text { Gland } \\
\text { Invasion } \\
\\
(n=41)\end{array}$ & $\begin{array}{l}\text { Metastatic } \\
\text { Mucosal SCC } \\
\text { to Parotid } \\
\text { Gland } \\
(n=3)\end{array}$ \\
\hline \multicolumn{5}{|l|}{ Sex } \\
\hline Females & 20 & 7 & 12 & 1 \\
\hline Males & 75 & 44 & 29 & 2 \\
\hline \multicolumn{5}{|l|}{ Race } \\
\hline White & 91 & 49 & 40 & 2 \\
\hline Black & 1 & - & 1 & - \\
\hline \multicolumn{5}{|l|}{ Age (in years) } \\
\hline Mean & 73.5 & 75.0 & 73.3 & 48 \\
\hline \multicolumn{5}{|l|}{ Tumor grade } \\
\hline Well differentiated & 14 & 7 & 7 & - \\
\hline Moderately differentiated & 35 & 15 & 19 & 1 \\
\hline Poorly differentiated & 46 & 29 & 15 & 2 \\
\hline \multicolumn{5}{|l|}{ Tumor keratinization } \\
\hline Keratinizing & 85 & 42 & 40 & 3 \\
\hline Non-keratinizing & 10 & 9 & 1 & 0 \\
\hline \multicolumn{5}{|l|}{ AJCC8 Group } \\
\hline II & 1 & - & 1 & - \\
\hline III & 29 & 9 & 20 & - \\
\hline IV & 65 & 42 & 20 & 3 \\
\hline \multicolumn{5}{|c|}{\begin{tabular}{|l|l|}
$\begin{array}{l}\text { Overall Patient Outcome (\#, average follow-up in } \\
\text { years) }\end{array}$ & \\
\end{tabular}} \\
\hline Alive no evidence of disease & $35(6.5)$ & $20(6.4)$ & $14(6.6)$ & $1(4.5)$ \\
\hline Alive with local disease & $1(6.7)$ & - & $1(6.7)$ & - \\
\hline Dead no evidence of disease & $15(4.1)$ & $11(4.1)$ & $4(3.9)$ & - \\
\hline Dead persistent disease (of another cause) & $5(1.4)$ & $2(0.7)$ & $2(1.4)$ & $1(2.8)$ \\
\hline Dead with disease & $39(1.4)$ & $18(1.8)$ & $20(1.1)$ & $1(1.8)$ \\
\hline
\end{tabular}

Conclusions: All cases of parotid gland SCC represent metastatic SCC (94\% cSCC; $6 \% \mathrm{mSCC})$ or cSCC with direct invasion into the gland. Metastatic SCC patients seem to have a better outcome overall, while tumors with direct extension are more likely to die from disease in a relatively short time (average $1.1 \mathrm{yrs}$ ). No primary SCC of the parotid gland were identified.

\section{Barnaculate Carcinoma: A Squamous Cell Carcinoma Type Seen in Patients with Proliferative Verrucous Leukoplakia: A Report of 14 Cases \\ Lester Thompson, Southern California Permanente Medical Group, Woodland Hills, CA}

Disclosures: Lester Thompson: None

Background: Proliferative verrucous leukoplakia (PVL) is an oral cavity disease that follows a relentless, but variable clinical course, often with carcinoma development. Certain associated squamous cell carcinomas (SCCs) are often bulky, with a stuck-on appearance. This study highlights features of barnaculate carcinoma. 
Design: Patients with at least 60 months of treatment duration and a clinical diagnosis of PVL were reviewed. Carcinoma type, time to development and management was evaluated.

Results: 19 patients met inclusion criteria, including 11 females and 8 males, with a median age at PVL diagnosis of 64 years (range 45-85). There were 10 ever smokers and 9 ever drinkers. Patients were managed over an average of 158 mos (range 61-353 mos) from first PVL diagnosis, with 17 patients developing carcinoma, an average of 61.2 mos after initial diagnosis (range $0-153$ mos). Six patients developed $>1$ carcinoma with a total of 29 carcinomas included. Five SCC types were observed (count of carcinomas): Barnaculate carcinoma $(n=14)$; conventional SCC (well [n=10] or moderately differentiated [n=3]); \& one each verrucous carcinoma and carcinoma cuniculatum. Barnaculate type appeared clinically as a stuck-on, bulky exophytic tumor. Histologically, voluminous, complex foldings and invaginations of exophytic and endophytic epithelium were present, lacking any significant extension below the level of the adjacent epithelium. Bulbous projections lacked significant pleomorphism and had limited mitoses. An inflammatory infiltrate, including intraepithelial microabscesses, blurred the interface, but destructive, single cell infiltration was absent. The surface was rounded with limited keratosis, lacking verrucous growth, church-spire type keratosis, and parakeratotic crypting. None of the barnaculate SCC cases (13 patients) developed lymph node metastasis. All patients were managed by wide or radical excision. Overall, 16 patients were alive with PVL (average 164 mos), a 100-mo average follow-up after carcinoma diagnosis. In the barnaculate carcinoma group: 10 patients were alive (average 153 mos) with 78 months mean follow-up after carcinoma diagnosis; 3 patients were dead at 77, 80, and $95 \mathrm{yrs}$, respectively, with persistent PVL (average 127 months), mean follow-up of 45 months after carcinoma diagnosis.

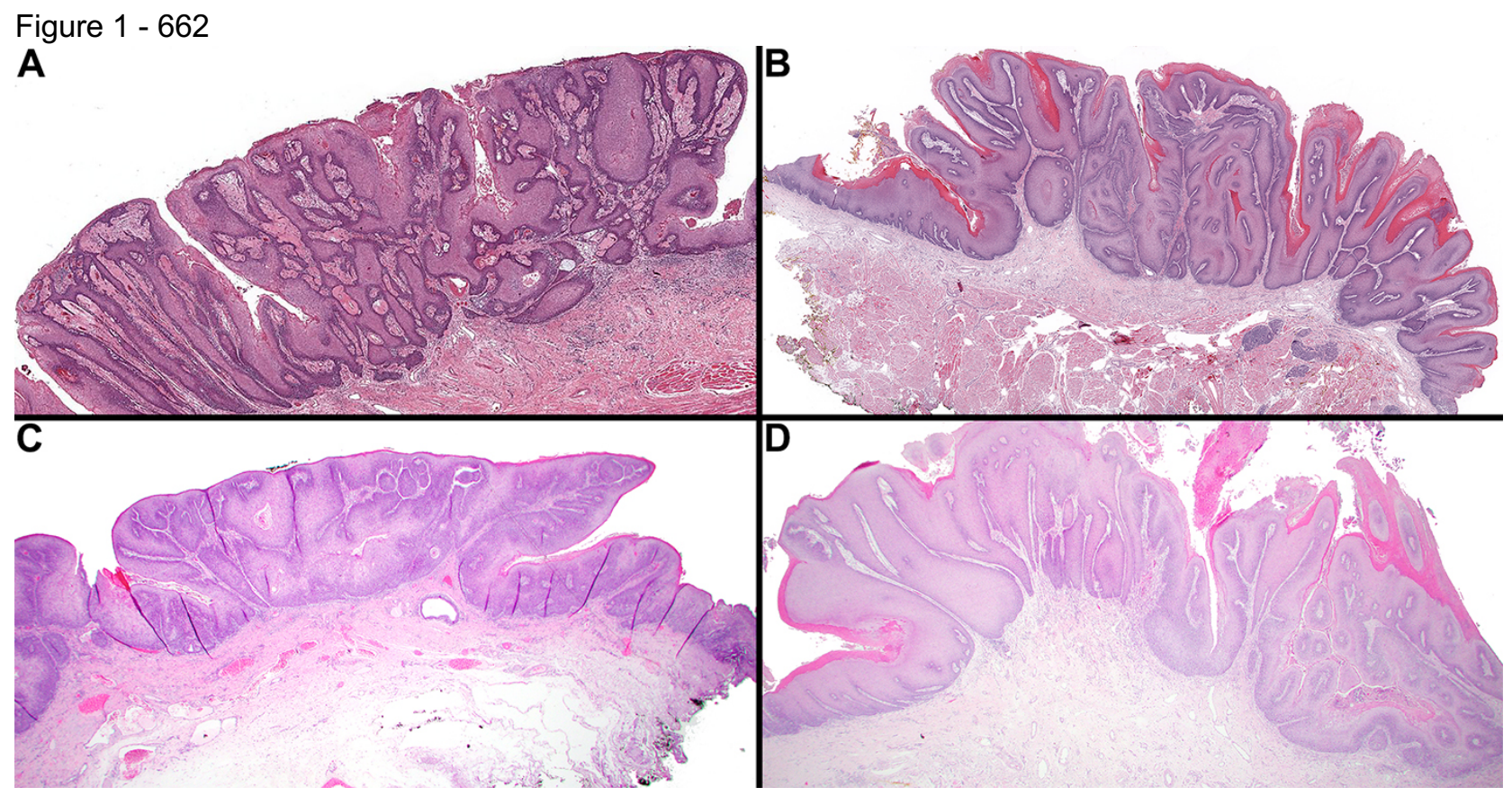

Conclusions: There is a very high risk of developing carcinoma when PVL patients are followed over a long period, recognizing there may be death with disease (average $84 \mathrm{yrs}$ ). In all of the barnaculate carcinoma cases, there were no lymph node metastases. These findings suggest conservative excision of barnaculate carcinoma cases without lymph node dissection may yield a lower morbidity in a PVL patient who will experience a long clinical course and potentially death with disease rather than death from disease. 


\section{Clinicopathologic Analysis of 71 Carcinomas ex Pleomorphic Adenoma}

Rumeal Whaley ${ }^{1}$, Bryan Hair², Emad Ababneh¹, Chandana Reddy², David Adelstein², Brian Burkey², Shlomo Koyfman ${ }^{2}$, Christopher Griffith ${ }^{1}$

${ }^{1}$ Cleveland Clinic Foundation, Cleveland, $\mathrm{OH},{ }^{2}$ Cleveland Clinic, Cleveland, $\mathrm{OH}$

Disclosures: Rumeal Whaley: None; Emad Ababneh: None; David Adelstein: None; Brian Burkey: None; Christopher Griffith: None

Background: Carcinoma ex pleomorphic adenoma (PA) is a rare neoplasm. Classical teaching states that any carcinoma of salivary origin can occur from a PA. Current molecular insights have improved our understanding of various salivary gland neoplasms. Mucoepidermoid carcinoma, adenoid cystic carcinoma, acinic cell carcinoma, and a variety of other neoplasms show recurrent genetic abnormalities. With this investigation of a large series carcinomas ex PA we show the relative propensities of certain carcinomas to develop from a PA and document their behaviors relative to one another.

Design: Carcinomas ex PA were retrieved from the authors' in-house archives. Seventy-one cases over a 21-year period (1999-2020) were identified. The clinicopathologic data was compiled and a retrospective review was undertaken.

Results: Follow up data was available for all cases. Forty-two (60\%) patients were female and mean age at first diagnosis was 63 years (range 26-92). Fifty-nine (83\%) cases arose in the parotid and the most common carcinomas were epithelial-myoepithelial carcinoma and salivary duct carcinoma (both $25 \%$; $n=18$ ), followed by myoepithelial carcinoma $(19.4 \%, n=14)$, and adenocarcinoma not otherwise specified (NOS) $(18.1 \%, n=13)$. Average tumor size was $3.47 \mathrm{~cm}$ (range $0.7-14.5)$. Sixty (84\%) were invasive (range 1-19 mm), eight cases (11.3\%) were non-invasive, and three cases $(4.2 \%)$ were invasive but the distance could not be determined. The majority were high-grade carcinomas $(63 \%, \mathrm{n}=45)$. Hyalinized PA was identified in half of the cases. Margins were either close $(<5 \mathrm{~mm})$ or positive in $63.9 \%$. Roughly two-thirds of patients had a lymph node dissection with one-third having metastatic disease. Forty-two patients were treated with radiation and 12 received chemotherapy. Mean follow up was 58 months (range 0-367). Eleven cases recurred locally, 5 regionally, and 13 distantly. Seventeen patients were dead at last follow up, 10 deaths were attributed to their carcinoma ( 3 epithelial-myoepithelial carcinoma, 3 adenocarcinoma NOS, 3 salivary duct carcinoma, 1 myoepithelial carcinoma). The non-invasive cases experience no locoregional recurrence nor metastatic disease (follow up average 84.6 months, range 6.7367).

Conclusions: We provide further evidence that non-invasive carcinoma ex PA follow an indolent clinical course. Interestingly, epithelial-myoepithelial carcinoma, salivary duct carcinoma, adenocarcinoma NOS occurred at similar rates and had similar rates of local, regional, and distance recurrences. Common salivary carcinomas such as mucoepidermoid carcinoma, adenoid cystic carcinoma, and acinic cell carcinoma are missing from this series as would be suggested by their differing molecular underpinnings. 